\title{
VARIATIONS ON INVERSION THEOREMS FOR NEWTON-PUISEUX SERIES
}

\author{
EVELIA ROSA GARCÍA BARROSO, PEDRO DANIEL GONZÁLEZ PÉREZ, AND PATRICK POPESCU-PAMPU
}

\begin{abstract}
Let $f(x, y)$ be an irreducible formal power series without constant term, over an algebraically closed field of characteristic zero. One may solve the equation $f(x, y)=0$ by choosing either $x$ or $y$ as independent variable, getting two finite sets of Newton-Puiseux series. In 1967 and 1968 respectively, Abhyankar and Zariski published proofs of an inversion theorem, expressing the characteristic exponents of one set of series in terms of those of the other set. In fact, a more general theorem, stated by Halphen in 1876 and proved by Stolz in 1879, relates also the coefficients of the characteristic terms of both sets of series. This theorem seems to have been completely forgotten. We give two new proofs of it and we generalize it to a theorem concerning irreducible series with an arbitrary number of variables.
\end{abstract}

This paper appeared online in Mathematische Annalen on 3.12.2016. The final publication is available at Springer via http://dx.doi.org/10.1007/s00208-016-1503-1

\section{CONTEnts}

1. Introduction

2. Newton-Puiseux series and their characteristic exponents

3. A calculus for the irreducible terms of invertible entire series

4. Applications to inversion formulae for Newton-Puiseux series

4.1. The first proof of the Halphen-Stolz theorem

4.2. The second proof of the Halphen-Stolz theorem

5. Generalization to an arbitrary number of variables

5.1. Irreducible exponents of subsets of $\mathbb{Q}^{h}$ with bounded denominators

5.2. On the notions of dual and reciprocal series in several variables

5.3. Newton-Puiseux series in several variables

5.4. The generalized Halphen-Stolz inversion theorem

5.5. The special case of quasi-ordinary series

References

\section{INTRODUCTION}

Let $f(x, y)$ be a polynomial with complex coefficients and without constant term. In his Treatise of fluxions and of infinite series [29, Sect. XXIX-XXXIII], Newton described an iterative method to compute a formal power series $\eta \in \mathbb{C}[[t]]$, such that $f\left(x, \eta\left(x^{1 / n}\right)\right)=0$ for certain positive integer $n$. In his 1850 paper [32, Puiseux proved that the series produced by Newton's algorithm were convergent whenever one starts from a convergent series $f(x, y) \in \mathbb{C}[[x, y]]$. Since then, the series whose exponents are positive rational numbers with bounded denominators, be they convergent or not, are called either Newton-Puiseux series or Puiseux series. In the sequel we will use the first denomination.

In the years 1870, Smith [34] and Halphen [20] realized that for several questions about the singularities of plane algebraic curves, a finite number of the exponents of a Newton-Puiseux series were more

Date: 3 December 2016

2010 Mathematics Subject Classification. 14B05 (primary), 14H20, 32S25.

Key words and phrases. Branch, Characteristic exponents, plane curve singularities, hypersurface singularities, quasiordinary series, Lagrange inversion, Newton-Puiseux series. 
important than the others. Halphen called those special exponents characteristic. Their modern definition is the following one: if one looks at the sequence of exponents taken in increasing size, then the characteristic ones are exactly those at which jumps the lowest common denominator which may be used for the exponents up to that point. It is a basic fact that if $f(x, y) \in \mathbb{C}[[x, y]]$ is irreducible, then all the associated Newton-Puiseux series have the same sequence of characteristic exponents. Let us call it the characteristic sequence of $f(x, y)$ relative to $x$.

Nowadays, one describes usually the importance of this notion as follows. Consider a branch $C$ (that is, an irreducible germ of curve) on a germ of smooth complex analytic surface $S$. If $(x, y)$ are local coordinates on $S$ and $f(x, y) \in \mathbb{C}[[x, y]]$ is a defining function of $C$ relative to those coordinates, then one may consider its characteristic sequence relative to $x$. This sequence is independent of $(x, y)$ once the $y$-axis is transversal to the branch $C$, that is, once its tangent does not coincide with the reduced tangent cone of $C$, which is a line. One speaks then of the generic characteristic sequence of $C$. Its main property is that it is a complete invariant of the embedded topological type of the branch in the ambient germ of surface (see for instance [38, Theorem 5.5.8]). In fact, most computations of other topological invariants of the pair $(S, C)$ are done in terms of its generic characteristic sequence.

It is nevertheless important to work also with Newton-Puiseux series computed relative to non-generic coordinate systems. For instance, another usual way to study the branch $C$ is to perform its process of embedded resolution by blow-ups of points (see for instance [38, Chapter 3]). A basic problem is then to express the generic characteristic sequence of the strict transform of $C$ obtained after one blow up in terms of that of $C$. If one starts from a generic Newton-Puiseux series $\eta\left(x^{1 / n}\right)$ of $C$, then $x^{-1} \cdot \eta\left(x^{1 / n}\right)$ is a Newton-Puiseux series of the strict transform of $C$. This series is generic for the strict transform if and only if the $x$-order of $\eta\left(x^{1 / n}\right)$ is at least 2 . In this case, it is immediate to get from it the generic characteristic sequence of the strict transform. But how to proceed when this is not the case?

One gets the following problem, in whose formulation we replaced for simplicity the strict transform by the initial branch: to compute the generic characteristic exponents of $C$ in terms of those of a NewtonPuiseux series $\eta\left(x^{1 / n}\right)$ of a defining function $f(x, y)$, when $C$ is tangent to the $y$-axis. But in this case $C$ is necessarily transversal to the $x$-axis. Therefore, if $\xi\left(y^{1 / m}\right)$ is a Newton-Puiseux series of $f(x, y)$ relative to $y$, that is, if $f\left(\xi\left(y^{1 / m}\right), y\right)=0$, then its characteristic sequence is exactly the generic characteristic sequence of $C$. Consequently, it is enough to express the characteristic sequence of $\xi\left(y^{1 / m}\right)$ in terms of that of $\eta\left(x^{1 / n}\right)$. Such an inversion theorem (called in this way because one inverts the roles of $x$ and $y$ in passing from $\eta\left(x^{1 / n}\right)$ to $\left.\xi\left(y^{1 / m}\right)\right)$ is well-known and it is often attributed to Abhyankar's paper 22 of 1967 or to Zariski's paper [40 of 1968. Proofs of this inversion theorem can be also found in [7, Section 5.6], [10, Theorem 5.2.21], [31, Proposition 4.3] and [3, Page 111].

We were very surprised to discover that in his 1876 paper [20, page 91], Halphen had already formulated a stronger result than the previous inversion theorem. He did not provide a proof of it. As far as we know, the first proof was given by Stolz [36, Sect. 3] in 1879. For this reason, we will speak in the sequel about the Halphen-Stolz inversion theorem. It is stronger than the inversion theorem of Abhyankar-Zariski because it does not only provide formulae for the characteristic exponents of $\xi\left(y^{1 / m}\right)$ in terms of those of $\eta\left(x^{1 / n}\right)$, but also for the corresponding coefficients. The previous papers of Halphen and Stolz seem to be forgotten, even though they were mentioned in Halphen's appendix [21] to Salmon's treatise on plane algebraic curves, which is cited sometimes nowadays.

The aim of our paper is to extend the Halphen-Stolz inversion theorem to an arbitrary number of variables. We achieve this aim in Corollary 5.21 of our Inversion Theorem 5.20,

In order to arrive at those results, we give first two new proofs of the classical Halphen-Stolz inversion theorem (stated by us as Corollary 4.5 of Theorem 4.4). The first one is based on the relations between the coefficients associated to the irreducible exponents of an invertible power series (see Definition 3.2), those of its powers and those of its dual (see Proposition 3.16). A flow-chart representing our line of reasoning for this first proof is drawn in diagram (4.17). Our second proof uses a formula expressing all the coefficients of the Newton-Puiseux series $\xi\left(y^{1 / m}\right)$ in terms of those of $\eta\left(x^{1 / n}\right)$ (see Proposition 4.10). This formula, based on the Lagrange inversion theorem (see Theorem 4.9), generalizes the Halphen-Stolz inversion formula for the coefficients. 
It is the first proof which we extend into a proof of the several-variables case. We could have given directly the most general statements and proofs. We preferred to start explaining in a detailed way the classical case, because it served us as a model for building the general proof, and because we feel that in this way the paper is easier to read. Its title is inspired by the title of Griffiths' paper [19.

As the generic characteristic sequence is crucial for understanding the embedded topology of complex plane branches, we expect that the associated coefficients could play an important role in problems related not only to their topology, but also to their analytical type. It is the main reason why we considered that it is important to bring to the attention of researchers the forgotten inversion theorem of Halphen-Stolz.

Zariski's proof of the inversion theorem in [40] was obtained as an application of the theory of saturation of local rings, in connexion with the study of topological equisingularity. There exist other notions of saturation, for instance, the Lipschitz-saturation (see [30, 25]) and also the presaturation of Campillo, which is better adapted to positive characteristic (see [5, 6]). In the case of irreducible germs of quasiordinary hypersurface singularities, Zariski's results on saturation and Lipman's inversion theorem appear also in the combinatorial characterization of the embedded topological type of this class of singularities (see [14, 27]). We expect that these lines of research combined with our generalized inversion theorem will lead to a better understanding of the invariants of singularities of other classes of hypersurface germs.

The article is structured as follows. In Section 2 we recall basic facts about Newton-Puiseux series associated to plane branches and their characteristic exponents. In Section 3 we introduce the notions of irreducible and essential exponents of a series and we give some results relating the coefficients of certain pairs of invertible series. In Section 4 we explain our proofs of the Halphen-Stolz inversion theorem and of its generalization into an inversion formula for all coefficients, based on a Lagrange inversion formula. Finally, in Section 5 we prove our generalization concerning an arbitrary number of variables and we explain in which way it extends the inversion theorem of Lipman concerning the characteristic exponents of quasi-ordinary series. Note that we work always over a fixed algebraically closed field of characteristic zero.

Acknowledgements. This research was partially supported by the French grants ANR-12-JS010002-01 SUSI, Labex CEMPI ANR-11-LABX-0007-01 and the Spanish grants MTM2012-36917-C03-0, MTM2013-45710-C2-2-P, MTM2016-76868-C2-1-P, MTM2016-80659-P. We are grateful to Herwig Hauser and Hana Kováčová for their translation of parts of Stolz' paper. The third-named author is grateful to Mickaël Matusinski for the opportunity to explain our first proof of the Halphen-Stolz theorem at the Geometry seminar of the University of Bordeaux. We thank him and the anonymous referee for their remarks on a previous version of this article, which allowed us to improve our presentation. We also thank Antonio Campillo for the information he sent us about the uses of Abhyankar-Zariski inversion.

\section{NEWTON-PUISEUX SERIES AND THEIR CHARACTERISTIC EXPONENTS}

In this section we introduce the notations and vocabulary about power series with integral or rational exponents which will be used throughout the text. Among the series with rational exponents, we will be interested only in those with bounded denominators, called Newton-Puiseux series. We conclude the section introducing the well-known notion of characteristic exponents of a Newton-Puiseux series.

Throughout the paper $\mathbb{N}$ denotes the set of non-negative integers, $\mathbb{N}^{*}$ denotes $\mathbb{N} \backslash\{0\}$ and $\mathbb{K}$ denotes a fixed algebraically closed field of characteristic zero.

The following definition explains the basic vocabulary and notations about power series with integer exponents which will be used in the sequel:

Definition 2.1. The ring $\mathbb{K}[[t]]$ of entire series consists of the formal power series in the variable $t$, with coefficients in $\mathbb{K}$ and exponents in $\mathbb{N}$. We say that the elements of its field of fractions $\mathbb{K}((t))$ are meromorphic series. They are exactly the series with coefficients in $\mathbb{K}$, exponents in $\mathbb{Z}$ and a finite number of terms with negative exponents. If $\eta \in \mathbb{K}((t))$ and $m \in \mathbb{Z}$, we denote by $[\eta]_{m} \in \mathbb{K}$ the coefficient of the monomial $t^{m}$ in $\eta(t)$ and by $\mathcal{S}(\eta) \subset \mathbb{Z}$ its support, consisting of the exponents $m$ with non-zero coefficients $[\eta]_{m}$. 
Therefore, a meromorphic series $\eta \in \mathbb{K}((t))$ may be written as:

$$
\eta(t)=\sum_{m \in \mathcal{S}(\eta)}[\eta]_{m} t^{m} .
$$

We will also use series with rational exponents, but such that their support has bounded denominators:

Definition 2.2. A Newton-Puiseux series $\psi$ in the variable $x$ is a power series of the form $\eta\left(x^{1 / n}\right)$, where $\eta(t) \in \mathbb{K}[[t]]$ and $n \in \mathbb{N}^{*}$. For a fixed $n \in \mathbb{N}^{*}$, they form the ring $\mathbb{K}\left[\left[x^{1 / n}\right]\right]$. Its field of fractions is denoted $\mathbb{K}\left(\left(x^{1 / n}\right)\right)$.

One extends immediately to Newton-Puiseux series $\psi$ the notion of support (which is a set with bounded denominators in the sense of Definition 3.1) and the notation $[\psi]_{m}$ for their coefficients (where now $m \in \mathbb{Q}_{+}$).

Denote by:

$$
\mathbb{K}\left[\left[x^{1 / \mathbb{N}}\right]\right]:=\bigcup_{n \in \mathbb{N}^{*}} \mathbb{K}\left[\left[x^{1 / n}\right]\right]
$$

the local $\mathbb{K}$-algebra of Newton-Puiseux series in the variable $x$.

The algebra $\mathbb{K}\left[\left[x^{1 / \mathbb{N}}\right]\right]$ is endowed with the natural order valuation:

$$
\operatorname{ord}_{x}: \mathbb{K}\left[\left[x^{1 / \mathbb{N}}\right]\right] \longrightarrow \mathbb{Q}_{+} \cup\{\infty\}
$$

which associates to each series $\psi=\eta\left(x^{1 / n}\right) \in \mathbb{K}\left[\left[x^{1 / n}\right]\right]$ the minimum of its support. The dominating coefficient of a Newton-Puiseux series $\psi$ is the coefficient of its term of exponent $\operatorname{ord}_{x}(\psi)$.

The field of fractions of the ring $\mathbb{K}\left[\left[x^{1 / \mathbb{N}}\right]\right]$ of Newton-Puiseux series is:

$$
\mathbb{K}\left(\left(x^{1 / \mathbb{N}}\right)\right):=\bigcup_{n \in \mathbb{N}^{*}} \mathbb{K}\left(\left(x^{1 / n}\right)\right) .
$$

One has the following fundamental theorem (see for instance, [10, Chapter 5.1], 13, Chapter 7], [9, Chapter 2.1] or [38, Chapter 2] for a proof), which explains the reason why we need to work with Newton-Puiseux series even if we are interested primarily in series with integral exponents:

Theorem 2.3 (The Newton-Puiseux theorem).

Any monic reduced polynomial $f \in \mathbb{K}[[x]][y]$ of degree $n \in \mathbb{N}^{*}$ has $n$ roots in $\mathbb{K}\left[\left[x^{1 / \mathbb{N}}\right]\right]$. If $f$ is moreover irreducible, then those roots are precisely the series of the form:

$$
\psi_{\rho}:=\eta\left(\rho \cdot x^{1 / n}\right),
$$

where $\psi=\eta\left(x^{1 / n}\right) \in \mathbb{K}\left[\left[x^{1 / n}\right]\right]$ is any one of them and $\rho \in \mathbb{K}^{*}$ varies among the multiplicative subgroup $G_{n}$ of $\left(\mathbb{K}^{*}, \cdot\right)$ of $n$-th roots of $1 \in \mathbb{K}^{*}$.

Remark 2.4. If $f \in \mathbb{K}[[x]][y]$ is a monic irreducible polynomial of degree $n \in \mathbb{N}^{*}$ and if $\psi \in \mathbb{K}\left(\left(x^{1 / n}\right)\right)$ is a root of $f$, then the field extension:

$$
\mathbb{K}((x)) \subset \mathbb{K}((x))[y] /(f) \simeq \mathbb{K}((x))[\psi]=\mathbb{K}\left(\left(x^{1 / n}\right)\right)
$$

is Galois. Its Galois group is isomorphic to the group $G_{n}$, acting on $\mathbb{K}\left(\left(x^{1 / n}\right)\right)$ by:

$$
\left(\rho, x^{1 / n}\right) \rightarrow \rho \cdot x^{1 / n}, \text { for all } \rho \in G_{n} .
$$

The series $\psi_{\rho}$ in Theorem 2.3 are precisely the conjugates of $\psi$ under this action.

Given a Newton-Puiseux series $\psi$, there exists an infinite number of choices of $n \in \mathbb{N}^{*}$ such that $\psi \in \mathbb{K}\left[\left[x^{1 / n}\right]\right]$. This is simply due to the fact that $\mathbb{K}\left[\left[x^{1 / n}\right]\right] \subset \mathbb{K}\left[\left[x^{1 / m}\right]\right]$ whenever $n$ divides $m$. One may get nevertheless a canonical choice of $n \in \mathbb{N}^{*}$ by asking it to be minimal:

Definition 2.5. If $\psi \in \mathbb{K}\left(\left(x^{1 / \mathbb{N}}\right)\right)$, a representation $\psi=\eta\left(x^{1 / n}\right)$ with $\eta(t) \in \mathbb{K}[[t]]$ and $n \in \mathbb{N}^{*}$ is called primitive if $n$ is the lowest common denominator of the exponents of $\psi$. 
Example 2.6. Assume that $\psi=x^{5 / 2}+x^{8 / 3}$. Then $\psi=\eta\left(x^{1 / 6}\right)$, with $\eta(t)=t^{15}+t^{16}$. This defines a primitive representation of $\psi$. Writing now $\psi=\eta_{1}\left(x^{1 / 12}\right)$ with $\eta_{1}(t)=t^{30}+t^{32}$, one gets a non-primitive representation. Let us consider a 6 -th root of unity $\rho \in \mathbb{K}^{*}$. Then:

$$
\psi_{\rho}=\eta\left(\rho x^{1 / 6}\right)=\rho^{15} x^{5 / 2}+\rho^{16} x^{8 / 3}=\rho^{3} x^{5 / 2}+\rho^{4} x^{8 / 3} .
$$

Among the exponents of a Newton-Puiseux series, several are distinguished by looking at the way they may be written as quotients of coprime integers:

Definition 2.7. Let $\psi \in \mathbb{K}\left[\left[x^{1 / \mathbb{N}}\right]\right]$ be a nonzero Newton-Puiseux series with zero constant term. The set $\mathcal{E}(\psi)$ of characteristic exponents of $\psi$ consists of the elements of the support of $\psi$ which, when written as quotients of integers, need a denominator strictly bigger than the lowest common denominator of the previous exponents. That is:

$$
\mathcal{E}(\psi):=\left\{l \in \mathcal{S}(\psi) \mid N_{l} \cdot l \notin \mathbb{Z}\right\}, \text { where } N_{l}:=\min \left\{N \in \mathbb{N}^{*} \mid(\mathcal{S}(\psi) \cap[0, l)) \subset \frac{1}{N} \mathbb{Z}\right\} .
$$

The sequence of characteristic exponents of $\psi \in \mathbb{K}\left[\left[x^{1 / \mathbb{N}}\right]\right]$ is defined by writing the elements of $\mathcal{E}(\psi)$ in increasing order.

Example 2.8. Both Newton-Puiseux series $x^{5 / 2}+x^{8 / 3}$ and $2 x-x^{5 / 2}+x^{8 / 3}-3 x^{7 / 2}+x^{23 / 6}$ have the same set $\{5 / 2,8 / 3\}$ of characteristic exponents.

Remark 2.9. According to Enriques and Chisini [11, page 375], it was Smith [34 and Halphen [20] who discovered in the years 1870 that special exponents of a Newton-Puiseux series are particularly important if one wants to compute the intersection number of two plane branches starting from their Newton-Puiseux series. This information was repeated by Zariski [39, Ch. 1], but without citing anymore their precise papers. Those special exponents were called characteristic by Halphen in [20, Sect. 1.1], which is also the paper in which he stated his inversion theorem for both exponents and coefficients. This denomination remained, but with slightly variable meanings (see also Remarks 2.12 and 4.8). Let us mention that Smith did not name those special exponents (which he defined in [34, Sect. 8]).

The set $\mathcal{E}(\psi)$ of characteristic exponents of $\psi$ is necessarily finite, even if the series has infinite support. More precisely, if $\psi \in \mathbb{K}\left[\left[x^{1 / n}\right]\right]$, then $\mathcal{E}(\psi)$ has at most as many elements as the number of factors of the prime factorisation of $n$. The set $\mathcal{E}(\psi)$ may also be characterized using the Galois action, as the set of orders $\operatorname{ord}_{x}\left(\psi_{\rho}-\psi\right.$ ), when $\rho$ varies in $G_{n} \backslash\{1\}$ (see for instance [38. Prop. 4.13]). Note that, because all the conjugates $\psi_{\rho}$ have the same support (by the explicit description of the Galois action recalled in Remark 2.4), they also have the same set of characteristic exponents, a fact implicitly used in Definition 2.11 below.

One has the following particular case of the Weierstrass preparation theorem (see for instance 10. Chap. 3.2] or [13, Chap. 6]):

Theorem 2.10. Let $f \in \mathbb{K}[[x, y]]$ be a series such that $\operatorname{ord}_{y}(f(0, y))=n \in \mathbb{N}^{*}$. Then, there exist a unique monic polynomial $F \in \mathbb{K}[[x]][y]$ of degree $n$ and a unique unit $\epsilon \in \mathbb{K}[[x, y]]$ such that:

$$
f=\epsilon \cdot F \text {. }
$$

In addition, $f$ is irreducible in $\mathbb{K}[[x, y]]$ if and only if the polynomial $F$ is irreducible in $\mathbb{K}[[x]][y]$.

This theorem allows us to introduce the following vocabulary:

Definition 2.11. Let $f \in \mathbb{K}[[x, y]]$ be an irreducible series such that $\operatorname{ord}_{y} f(0, y)=n \in \mathbb{N}^{*}$. The polynomial $F \in \mathbb{K}[[x]][y]$ provided by Theorem 2.10 is called the Weierstrass polynomial associated to the series $f \in \mathbb{K}[[x, y]]$ relative to $x$. Then, the Newton-Puiseux series of $f$ relative to $x$ are the roots of the associated Weierstrass polynomial $F \in \mathbb{K}[[x]][y]$, in the $\operatorname{ring} \mathbb{K}\left[\left[x^{1 / \mathbb{N}}\right]\right]$. If $f$ is irreducible, then its characteristic exponents relative to $x$ are the characteristic exponents of any one of those roots. 
Remark 2.12. Let us explain how the previous algebraic notions apply in the geometrical setting of a branch, an irreducible germ of complex analytic plane curve $C$ on a germ $S$ of smooth complex analytic surface. Choose a local system of coordinates $(x, y)$ on $S$. Then, the branch $C$ is defined by an irreducible series $f \in \mathbb{C}[[x, y]]$. If the $y$-axis is transversal to the tangent line of the branch $C$, then one may show that the characteristic exponents of $f$ relative to $x$ are always the same (see for instance [38, Thm. 3.5.6]). One speaks in this case about generic characteristic exponents. The notations used for them by Zariski in [39, Ch. 1], 40, Sect. 3] and [41, Sect. II.3] are common nowadays: $\left(\frac{m_{1}}{n_{1}}, \frac{m_{2}}{n_{1} n_{2}}, \ldots, \frac{m_{g}}{n_{1} \cdots n_{g}}\right)$. At least since [40. Sect. 3], Zariski uses also a characteristic sequence $\left(\beta_{0}, \beta_{1}, \ldots, \beta_{g}\right)$ of natural numbers instead of the sequence of generic characteristic exponents, which may then be reconstructed as $\left(\frac{\beta_{1}}{\beta_{0}}, \ldots, \frac{\beta_{g}}{\beta_{0}}\right)$ (here $\beta_{0}$ is the multiplicity of the branch $C$, that is, the minimal degree of the monomials of $f(x, y))$. We do not use the previous notations in this paper for two reasons: on one side we never need a genericity hypothesis on the coordinate system relative to $C$ and on the other side we find the related notion of essential exponent relative to 1 (see Definition [3.6) better suited to a simple formulation of the Halphen-Stolz inversion theorem (see Remark 4.7).

\section{A CAlculus for the irReducible terms of INVERTible Entire SERIES}

In this section we introduce several notions allowing to study the supports of Newton-Puiseux series and the semigroups generated by them: their irreducible elements (see Definition 3.2) and their essential elements (see Definition 3.6) relative to an arbitrary natural number. We concentrate then on the entire series with non-zero constant terms. If $\phi$ is such a series, we introduce its dual $\check{\phi}$ and we show that the coefficients of the monomials with irreducible exponents in the positive integral powers of $\phi$ and in the dual $\breve{\phi}$ may be deduced from those of $\phi$ by simple formulae (see Proposition [3.16). In Section 4 we will apply those formulae to the essential exponents relative to well-chosen natural numbers, in order to prove the Halphen-Stolz inversion theorem.

Next definition introduces vocabulary about the sets of rational numbers which may appear as supports of Newton-Puiseux series:

Definition 3.1. A set with bounded denominators is a non-empty (possibly infinite) set $E \subset \mathbb{Q}$ such that there exists $n \in \mathbb{N}^{*}$ with $E \subset \frac{1}{n} \mathbb{N}$. We denote by $\mathbb{N}^{*} E \subset \mathbb{Q}_{+}$the semigroup generated by $E$, that is, the set of non-empty finite sums of elements of $E$. Analogously, we denote by $\mathbb{Z} E \subset \mathbb{Q}$ the group generated by $E$.

Note that the semigroup $\mathbb{N}^{*} E$ contains 0 (that is, it is a monoid for addition) if and only if $E$ does.

Given a set with bounded denominators, we will be interested in its irreducible elements:

Definition 3.2. Assume that $E \subset \mathbb{Q}_{+}$is a set with bounded denominators. We denote by $\operatorname{Irr}(E)$ the set of irreducible elements of $E$, that is, the subset of $E$ formed by those elements which cannot be written as sums of at least two elements of $E \backslash\{0\}$. The elements of $E$ which are not irreducible are called reducible. If $E$ is the support $\mathcal{S}(\psi)$ of a Newton-Puiseux series $\psi$, then we write also $\operatorname{Irr}(\psi):=\operatorname{Irr}(\mathcal{S}(\psi))$, and we call it the set of irreducible exponents of $\psi$.

Remark 3.3. Let $E \subset \mathbb{Q}_{+}$be a set with bounded denominators. Notice that if $E$ contains 0 , then 0 is by definition an irreducible element of $E$. More generally, the minimum of $E$ is always irreducible in $E$.

Example 3.4. Assume that $E=\{6,15,16,21,23\}$. Then $\operatorname{Irr}(E)=\{6,15,16,23\}$. Note that 21 is reducible in $E$, because it is equal to the sum $6+15$ and $6,15 \in E$.

The sets of irreducible elements of $E$ and of the semigroup it generates coincide:

Lemma 3.5. Assume that $E \subset \mathbb{Q}_{+}$is a set with bounded denominators. Then:

(1) $\operatorname{Irr}(E)=\operatorname{Irr}\left(\mathbb{N}^{*} E\right)$ and this set is the minimal generating set of the semigroup $\mathbb{N}^{*} E$, relative to the inclusion partial order on the set of its generating sets.

(2) The set $\operatorname{Irr}(E)$ is finite. 
Proof. Multiplying $E$ by a convenient rational number, we may restrict to the sets $E \subset \mathbb{N}$ whose elements are globally coprime.

(1) Both inclusions between $\operatorname{Irr}(E)$ and $\operatorname{Irr}\left(\mathbb{N}^{*} E\right)$ are immediate to check, therefore we will assume from now on that the two sets are equal.

Let us prove the minimality property of $\operatorname{Irr}\left(\mathbb{N}^{*} E\right)$. Consider another generating set $A$ of $\mathbb{N}^{*} E$ and $a \in \operatorname{Irr}\left(\mathbb{N}^{*} E\right)$. As $A$ is generating, $a$ may be written as a sum of elements of $A$. If this sum were non-trivial, then $a$ would not be irreducible in $\mathbb{N}^{*} E$. Therefore $a \in A$, which shows the desired inclusion $\operatorname{Irr}\left(\mathbb{N}^{*} E\right) \subset A$.

(2) In order to show that $\operatorname{Irr}(E)$ is finite, it is enough to show that $\operatorname{Irr}\left(\mathbb{N}^{*} E\right)$ is finite. The semigroup $\mathbb{N}^{*} E$ being generated by globally coprime elements, it has finite conductor, that is, there exists $c \in \mathbb{N}$ such that all natural numbers greater than or equal to $c$ belong to $\mathbb{N}^{*} E$ (see 38, page 82]; in this case, the smallest such $c$ is called the conductor of the numerical semigroup $\mathbb{N}^{*} E$ ). But this implies that $\operatorname{Irr}\left(\mathbb{N}^{*} E\right) \subset\{0,1, \ldots, 2 c-1\}$. Indeed, any element $l \geqslant 2 c$ of the semigroup may be written in the form $c+d$, with $d \geqslant c$, that is, as a non-trivial sum of elements of the semigroup.

We will be especially interested in particular sequences of irreducible elements of a given set $E$ with bounded denominators:

Definition 3.6. Let us consider a set $E \subset \mathbb{Q}_{+}$with bounded denominators and an integer $p \in \mathbb{N}^{*}$. Then the sequence $\operatorname{ess}(E, p):=\left(\operatorname{ess}(E, p)_{l}\right)_{l}$ of essential elements of $E$ relative to $p$ is defined inductively by:

- $\operatorname{ess}(E, p)_{0}:=\min E$.

- If $l \geqslant 1$, then the term $\operatorname{ess}(E, p)_{l}$ is defined if and only if $E$ is not included in the group $\mathbb{Z}\left\{p, \operatorname{ess}(E, p)_{0}, \ldots, \operatorname{ess}(E, p)_{l-1}\right\}$. In this case:

$$
\operatorname{ess}(E, p)_{l}:=\min \left(E \backslash \mathbb{Z}\left\{p, \operatorname{ess}(E, p)_{0}, \ldots, \operatorname{ess}(E, p)_{l-1}\right\}\right) .
$$

The following basic property of this notion is a direct consequence of the definition:

Lemma 3.7. Assume that $E \subset \mathbb{Q}_{+}$has bounded denominators and take $p, q \in \mathbb{N}^{*}$. Then:

$$
q \operatorname{ess}(E, p)=\operatorname{ess}(q E, q p) \text {. }
$$

One has also:

Lemma 3.8. Assume that $E \subset \mathbb{Q}_{+}$has bounded denominators and that $p \in \mathbb{N}^{*}$. Then the sequence of essential exponents $\operatorname{ess}(E, p)$ is finite.

Proof. Lemma 3.7 implies that it is enough to consider the case where $E \subset \mathbb{Z}$. Then one has by definition the strict inclusions:

$$
\mathbb{Z}\left\{p, \operatorname{ess}(E, p)_{0}, \ldots, \operatorname{ess}(E, p)_{l-1}\right\} \subsetneq \mathbb{Z}\left\{p, \operatorname{ess}(E, p)_{0}, \ldots, \operatorname{ess}(E, p)_{l}\right\}, \text { for all } l \geqslant 1
$$

(for which the term $\operatorname{ess}(E, p)_{l}$ is defined). Any ascending chain of subgroups of $\mathbb{Z}$ being stationary, we deduce that the sequence of essential exponents is finite.

Example 3.9. Let us consider again the set $E=\{6,15,16,21,23\}$ from Example 3.4. Here are its sequences of essential elements relative to the numbers $p \in\{1, \ldots, 12\}$ :

$$
\left\{\begin{array}{l}
\operatorname{ess}(E, 1)=\operatorname{ess}(E, 5)=\operatorname{ess}(E, 7)=\operatorname{ess}(E, 11)=(6), \\
\operatorname{ess}(E, 2)=\operatorname{ess}(E, 4)=\operatorname{ess}(E, 8)=\operatorname{ess}(E, 10)=(6,15), \\
\operatorname{ess}(E, 3)=\operatorname{ess}(E, 9)=(6,16), \\
\operatorname{ess}(E, 6)=\operatorname{ess}(E, 12)=(6,15,16) .
\end{array}\right.
$$

Lemma 3.7 implies that: ess $\left(\left\{1, \frac{5}{2}, \frac{8}{3}, \frac{7}{2}, \frac{23}{6}\right\}, 1\right)=\frac{1}{6} \operatorname{ess}(E, 6)=\left(1, \frac{5}{2}, \frac{8}{3}\right)$. The set $\left\{1, \frac{5}{2}, \frac{8}{3}, \frac{7}{2}, \frac{23}{6}\right\}$ is precisely the support of the second series considered in Example 2.8, whose sequence of characteristic exponents is $\left(\frac{5}{2}, \frac{8}{3}\right)$. Note that its sequence of essential exponents relative to 1 may be obtained from the characteristic sequence by adjoining to it as initial term the order of this series (which is in this case equal to 1). We will see in Lemma 3.13 that this is a general fact. 
The following lemma shows that the non-zero essential elements of a set $E$ relative to any positive integer are irreducible elements of $E$ :

Lemma 3.10. The essential elements of a set $E \subset \mathbb{Q}_{+}$with bounded denominators relative to a number $p \in \mathbb{N}^{*}$ are irreducible elements of $E$.

Proof. If $\operatorname{ess}(E, p)=\left(\epsilon_{0}, \ldots, \epsilon_{d}\right)$, then we have that $\epsilon_{0}=\min E$, which is an irreducible element of $E$.

Let us show that the property is also true for $\epsilon_{l}$, where $l \geqslant 1$. If $\epsilon_{l}$ was reducible, then it would be a non-trivial sum of elements of $E$, which would therefore be strictly less than $\epsilon_{l}$. By the definition of $\epsilon_{l}$, the terms of this sum would belong to the group $\mathbb{Z}\left\{p, \epsilon_{0}, \ldots, \epsilon_{l-1}\right\}$. This would imply that $\epsilon_{l}$ belongs also to this group, which contradicts Definition 3.6.

Lemma 3.11. Assume that $E \subset \mathbb{Q}_{+}$has bounded denominators and that $p \in \mathbb{N}^{*}$. Then we have the following equality of essential sequences:

$$
\operatorname{ess}(E, p)=\operatorname{ess}(\operatorname{Irr}(E), p) .
$$

Proof. If $\operatorname{ess}(E, p)=\left(\epsilon_{0}, \ldots, \epsilon_{d}\right)$ and $\operatorname{ess}(\operatorname{Irr}(E), p)=\left(\epsilon_{0}^{\prime}, \ldots, \epsilon_{d^{\prime}}^{\prime}\right)$, then Remark 3.3 and Definition 3.6 imply that:

$$
\epsilon_{0}=\min E=\min \operatorname{Irr}(E)=\epsilon_{0}^{\prime} .
$$

Assume by induction that $\epsilon_{0}=\epsilon_{0}^{\prime}, \ldots, \epsilon_{l-1}=\epsilon_{l-1}^{\prime}$, for $1 \leqslant l<d$. Then we get:

$$
\epsilon_{l}^{\prime}=\min \left(\operatorname{Irr}(E) \backslash \mathbb{Z}\left\{p, \epsilon_{0}, \ldots, \epsilon_{l-1}\right\}\right) \leqslant \epsilon_{l}=\min \left(E \backslash \mathbb{Z}\left\{p, \epsilon_{0}, \ldots, \epsilon_{l-1}\right\}\right),
$$

since $\epsilon_{l}$ is an irreducible element of $E$ by Lemma 3.10. The inclusion $\operatorname{Irr}(E) \subset E$ implies also that $\epsilon_{l} \leqslant \epsilon_{l}^{\prime}$, hence $\epsilon_{l}=\epsilon_{l}^{\prime}$. This proves that $\epsilon_{l}=\epsilon_{l}^{\prime}$ for $0 \leqslant l \leqslant d$ and $d \leqslant d^{\prime}$. By Lemma 3.10 and the definition of the essential exponents, one has the inclusions $\operatorname{Irr}(E) \subset E \subset \mathbb{Z}\left\{p, \epsilon_{0}, \ldots, \epsilon_{d}\right\}$, which imply that $d^{\prime}=d$.

Definition 3.12. If $\psi \in \mathbb{K}\left[\left[x^{1 / n}\right]\right]$ is a non-zero series and $p \in \mathbb{N}^{*}$, then we will write:

$$
\operatorname{ess}(\psi, p):=\operatorname{ess}(\mathcal{S}(\psi), p)
$$

and we will speak about the sequence of essential exponents of $\psi$ relative to $p$.

The characteristic exponents of a Newton-Puiseux series are intimately related to its essential exponents relative to 1 :

Lemma 3.13. Let $\left(\alpha_{1}, \ldots, \alpha_{g}\right)$ be the sequence of characteristic exponents of a series $\psi \in \mathbb{K}\left[\left[x^{1 / n}\right]\right]$. It may be obtained from the sequence $\left(\epsilon_{0}, \epsilon_{1}, \ldots, \epsilon_{d}\right)$ of essential exponents of $\psi$ relative to 1 in the following way:

- If $\epsilon_{0} \notin \mathbb{Z}$, then $g=d+1$ and $\alpha_{i}=\epsilon_{i-1}$ for all $i \in\{1, \ldots, d+1\}$.

- If $\epsilon_{0} \in \mathbb{Z}$, then $g=d$ and $\alpha_{i}=\epsilon_{i}$ for all $i \in\{1, \ldots, d\}$.

Proof.

- Consider first the case in which $\epsilon_{0} \notin \mathbb{Z}$. As the first characteristic exponent is the minimal nonintegral exponent in the support of $\psi$, we deduce that $\alpha_{1}=\epsilon_{0}$. Assume by induction that $\alpha_{i}=\epsilon_{i-1}$ for all $i \in\{0, \ldots, l\}$. Definition 3.6 implies that $\epsilon_{l}$ is the first exponent of $\mathcal{S}(\psi)$ which is strictly greater than $\epsilon_{l-1}$ and which cannot be written as a fraction whose denominator is the least common denominator of the previous exponents in the support of $\psi$. By Definition 2.7 we get that $\alpha_{l+1}=\epsilon_{l}$.

- Consider now the case in which $\epsilon_{0} \in \mathbb{Z}$. By definition, $\epsilon_{0} \in \mathbb{N}$ cannot be a characteristic exponent of $\psi$ and $\alpha_{1}=\epsilon_{1}$. The result follows by induction, using the same argument as in the first case.

In the rest of this section we will be especially interested in entire series with non-zero constant term, that is, in invertible elements of the multiplicative monoid $(\mathbb{K}[[t]], \cdot)$. They form a multiplicative group, which we will denote by $\left(\mathbb{K}[[t]]^{*}, \cdot\right)$.

Note that the entire series of the form $t \phi(t)$ for $\phi \in \mathbb{K}[[t]]^{*}$, that is, the entire series of order 1 , form the (non-commutative) group under composition of series which admit a reciprocal (an inverse for composition). We denote by $\left(t \mathbb{K}[[t]]^{*}, \circ\right)$ this group. Division by $t$ transforms it bijectively into $\left(\mathbb{K}[[t]]^{*}, \cdot\right)$, but is not a morphism of groups. What is essential for us is that the inversion for composition 
becomes an involution of the set $\mathbb{K}[[t]]^{*}$ which has special properties with respect to the terms whose exponents are irreducible (see Proposition 3.16 (2) ). We use the following vocabulary for this involution:

Definition 3.14. If $\phi \in \mathbb{K}[[t]]^{*}$, then its dual is the unique entire series $\breve{\phi} \in \mathbb{K}[[u]]^{*}$ such that $u \breve{\phi}(u)$ and $t \phi(t)$ are reciprocal.

Remark 3.15. If $\phi \in \mathbb{K}[[t]]^{*}$, then setting $u=t \phi(t)$ defines a change of variable in the ring $\mathbb{K}[[t]]$. Notice that $\mathbb{K}[[t]]=\mathbb{K}[[u]]$ and by Definition 3.14 the following equivalence holds:

$$
u=t \phi(t) \Leftrightarrow t=u \check{\phi}(u) \text {. }
$$

We use two variables $t$ and $u$ in our notations for a dual pair of series, in order to relate them easily from the notational point of view to the two sets of Newton-Puiseux series of an irreducible $f(x, y) \in \mathbb{K}[[x, y]]$, which depend on the two variables $x$ and $y$ (see Section 4).

The following proposition expresses the coefficients of the positive integral powers and of the dual of an entire series $\phi \in \mathbb{K}[[t]]^{*}$ in terms of those of $\phi$. It may be deduced from the statement and the proof of Wall's [38, Lemma 3.5.4]. But as we could not find it formulated in the literature and as it lies at the core of our first proof of the Halphen-Stolz theorem, we give a detailed proof of it.

Proposition 3.16. Let $\phi \in \mathbb{K}[[t]]^{*}$ and $N \in \mathbb{N}^{*}$. Then:

(1) $\operatorname{Irr}\left(\phi^{N}\right)=\operatorname{Irr}(\phi)$. Moreover $\left[\phi^{N}\right]_{0}=[\phi]_{0}^{N}$ and $\left[\phi^{N}\right]_{r}=N[\phi]_{0}^{N-1}[\phi]_{r}$, for all $r \in \operatorname{Irr}(\phi) \backslash\{0\}$.

(2) $\operatorname{Irr}(\breve{\phi})=\operatorname{Irr}(\phi)$. Moreover $[\check{\phi}]_{0}=[\phi]_{0}^{-1}$ and $[\check{\phi}]_{r}=-[\phi]_{0}^{-r-2}[\phi]_{r}$, for all $r \in \operatorname{Irr}(\phi) \backslash\{0\}$.

Proof. One has:

$$
\phi(t)=\sum_{j \in \mathcal{S}(\phi)}[\phi]_{j} t^{j} .
$$

The hypothesis $\phi \in \mathbb{K}[[t]]^{*}$ translates into $0 \in \mathcal{S}(\phi)$, that is, $[\phi]_{0} \neq 0$.

(1) Consider first the case of $\phi^{N}$. By equation (3.2), we have:

$$
\phi^{N}(t)=\sum_{j_{1}, \ldots, j_{N} \in \mathcal{S}(\phi)}[\phi]_{j_{1}} \cdots[\phi]_{j_{N}} t^{j_{1}+\cdots+j_{N}} .
$$

- Let us show first that $\operatorname{Irr}(\phi) \subset \operatorname{Irr}\left(\phi^{N}\right)$ and that one has the stated equalities between coefficients. Consider $r \in \operatorname{Irr}(\phi)$. If $r=0$, one has obviously $r \in \operatorname{Irr}\left(\phi^{N}\right)$ and $\left[\phi^{N}\right]_{0}=[\phi]_{0}^{N}$. Assume therefore that $r>0$. The only way to write $r$ as a sum of $N$ elements of $\mathcal{S}(\phi)$, is that one of them be equal to $r$, and the other ones vanish. There are $N$ different positions in the sum for the non-vanishing one, therefore:

$$
\left[\phi^{N}\right]_{r}=N[\phi]_{0}^{N-1}[\phi]_{r},
$$

which is the desired formula.

In particular, $\left[\phi^{N}\right]_{r} \neq 0$, which shows that $r \in \mathcal{S}\left(\phi^{N}\right)$. If $r$ was reducible in $\mathcal{S}\left(\phi^{N}\right)$, it could be written as a non-trivial sum of elements of $\mathcal{S}\left(\phi^{N}\right)$. By formula (3.3), it would also be a non-trivial sum of elements of $\mathcal{S}(\phi)$, which would contradict the fact that it is an irreducible element of $\mathcal{S}(\phi)$. Therefore $r \in \operatorname{Irr}\left(\phi^{N}\right)$.

- Let us show now the reverse inclusion $\operatorname{Irr}\left(\phi^{N}\right) \subset \operatorname{Irr}(\phi)$. Consider an element $r \in \operatorname{Irr}\left(\phi^{N}\right)$. By formula (3.3), we know that it may be written as a sum of $N$ elements of $\mathcal{S}(\phi)$. In particular, it may be written as a sum of irreducible elements of $\mathcal{S}(\phi)$. By the previous point, we know that those elements are also irreducible in $\mathcal{S}\left(\phi^{N}\right)$. Our hypothesis $r \in \operatorname{Irr}\left(\phi^{N}\right)$ implies that there is only one non-zero term in this sum, which proves the desired membership $r \in \operatorname{Irr}(\phi)$.

(2) Consider now the case of $\breve{\phi}$. Write the analogue of (3.2) for $\breve{\phi}$ :

$$
\breve{\phi}(u)=\sum_{k \in \mathcal{S}(\breve{\phi})}[\breve{\phi}]_{k} u^{k} \text {. }
$$

As $t \phi(t) \in \mathbb{K}[[t]]$ and $u \breve{\phi}(u) \in \mathbb{K}[[u]]$ are reciprocal series, one has by definition the identity:

$$
t=(t \phi(t)) \check{\phi}(t \phi(t)),
$$


which, after division by $t$ and combination with the expansion (3.4), gives:

$$
1=\sum_{k \in \mathcal{S}(\check{\phi})}[\check{\phi}]_{k} t^{k} \phi(t)^{k+1} \text {. }
$$

Expand now the powers $\phi(t)^{k+1}$ using equation (3.3). We get:

$$
1=\sum_{\substack{k \in \mathcal{S}(\check{\phi}) \\ j_{1}, \ldots, j_{k+1} \in \mathcal{S}(\phi)}}[\check{\phi}]_{k}[\phi]_{j_{1}} \cdots[\phi]_{j_{k+1}} t^{k+j_{1}+\cdots+j_{k+1}} .
$$

Therefore:

$$
\sum_{\substack{k \in \mathcal{S}(\check{\phi}) \\ j_{1}, \ldots, j_{k+1} \in \mathcal{S}(\phi) \\ k+j_{1}+\cdots+j_{k+1}=p}}[\check{\phi}]_{k}[\phi]_{j_{1}} \cdots[\phi]_{j_{k+1}}=0 \text {, for all } p \in \mathbb{N}^{*} .
$$

- Let us show first that $\operatorname{Irr}(\phi)=\operatorname{Irr}(\check{\phi})$. By Lemma 3.5 (1), the irreducible elements of a set are determined by the semigroup it generates. In order to show that the sets $\operatorname{Irr}(\phi)$ and $\operatorname{Irr}(\check{\phi})$ coincide, it is therefore enough to prove that:

$$
\mathbb{N}^{*}(\mathcal{S}(\phi))=\mathbb{N}^{*}(\mathcal{S}(\check{\phi})) .
$$

The situation being symmetric between $\phi$ and $\check{\phi}$, we may prove only the inclusion:

$$
\mathbb{N}^{*}(\mathcal{S}(\check{\phi})) \subset \mathbb{N}^{*}(\mathcal{S}(\phi)) .
$$

We will argue by contradiction, assuming that the previous inclusion is false. Consider then:

$$
r \in \mathbb{N}^{*}(\mathcal{S}(\check{\phi})) \backslash \mathbb{N}^{*}(\mathcal{S}(\phi)),
$$

which is minimal with this property. As $0 \in \mathcal{S}(\phi)$, we have $r>0$.

Apply equation (3.6) to $p=r$. Consider a tuple:

$$
\left(k, j_{1}, \ldots, j_{k+1}\right) \in \mathcal{S}(\check{\phi}) \times \mathcal{S}(\phi)^{k+1}
$$

such that:

$$
k+j_{1}+\cdots+j_{k+1}=r .
$$

Let us show that this implies the equality $k=r$. Reasoning again by contradiction, assume that $k<r$. As $k \in \mathcal{S}(\check{\phi}) \subset \mathbb{N}^{*}(\mathcal{S}(\check{\phi}))$, the minimality of $r$ shows that $k \in \mathbb{N}^{*}(\mathcal{S}(\phi))$. Combining condition (3.10) and equation (3.11), we deduce that $r \in \mathbb{N}^{*}(\mathcal{S}(\phi))$, which contradicts the assumption (3.9).

Therefore, if both (3.10) and (3.11) are true, then $k=r$, which implies that $j_{1}=\cdots=j_{r+1}=0$. Hence there is only one term in the sum of the left-hand side of equation (3.6) for $p=r$, and we get:

$$
[\check{\phi}]_{r}[\phi]_{0}^{r+1}=0,
$$

which contradicts the assumption that both coefficients $[\check{\phi}]_{r}$ and $[\phi]_{0}$ are non-zero (as they are associated to elements of the supports of $\phi$ and $\check{\phi}$ ).

Our proof of the inclusion (3.8) is finished. Therefore, as explained above, we get the desired equality $\operatorname{Irr}(\check{\phi})=\operatorname{Irr}(\phi)$.

- Let us prove the identities relating the coefficients associated to the irreducible exponents of $\phi$ and $\check{\phi}$. Consider $r \in \operatorname{Irr}(\phi)=\operatorname{Irr}(\check{\phi})$. Look again at the tuples $\left(k, j_{1}, \ldots, j_{k+1}\right)$ satisfying the conditions (3.10) and (3.11) above.

If $k \notin\{0, r\}$, then at least one of the numbers $j_{1}, \ldots, j_{k+1}$ would not vanish. Equation (3.11) gives a non-trivial decomposition of $r$ inside $\mathcal{S}(\phi) \cup \mathcal{S}(\check{\phi}) \subset \mathbb{N}^{*}(\mathcal{S}(\phi)) \stackrel{\sqrt[3.7]{=}}{=} \mathbb{N}^{*}(\mathcal{S}(\check{\phi}))$, which shows that $r \notin$ $\operatorname{Irr}\left(\mathbb{N}^{*} \mathcal{S}(\phi)\right)$. This contradicts Lemma 3.5 (1). 
Therefore, one has necessarily $k=0$ or $k=r$. Both possibilities determine completely $\left(j_{1}, \ldots, j_{k+1}\right)$ through equation (3.11). Applying equation (3.6) to $p=r$, we get:

$$
[\check{\phi}]_{0}[\phi]_{r}+[\check{\phi}]_{r}[\phi]_{0}^{r+1}=0 .
$$

The equalities (3.12) and $[\check{\phi}]_{0}=[\phi]_{0}^{-1}$ imply the formula for $[\check{\phi}]_{r}$ written in the statement of the proposition.

Combining Proposition 3.16 with Lemma 3.11, we get:

Corollary 3.17. Let $\phi \in \mathbb{K}[[t]]^{*}$ and $N, p \in \mathbb{N}^{*}$. Then the sequences of essential exponents of $\phi, \phi^{N}$ and $\check{\phi}$ relative to $p$ coincide.

In the next section we apply Proposition 3.16 and its Corollary 3.17 in order to relate the essential exponents relative to 1 and their coefficients for the Newton-Puiseux series of an irreducible series $f(x, y) \in$ $\mathbb{K}[[x, y]]$

\section{Applications to inversion formulae for Newton-Puiseux Series}

Let $f(x, y) \in \mathbb{K}[[x, y]]$ be an irreducible formal power series. One has therefore associated NewtonPuiseux series relative to both coordinates $x$ and $y$. In this section we prove in two ways the Halphen-Stolz theorem (Corollary 4.5), which relates the coefficients of the terms with essential exponents relative to 1 in both series. The first proof, summarized in the flow-chart (4.17), applies directly the results of the previous section. The second one passes through a more general result, allowing to compute recursively all the coefficients of one series in terms of those of the other one (see Proposition 4.10). In turn, this proposition is a consequence of a version of the classical Lagrange inversion formula (see Theorem 4.9).

\subsection{The first proof of the Halphen-Stolz theorem.}

There is no natural bijection between the Newton-Puiseux series of a formal power series $f(x, y)$ relative to $x$ and $y$, for the simple reason that their numbers are in general different. We want to explain first that if one takes adequate roots of them, then one gets two sets which are naturally in a bijective correspondence (see Proposition 4.2).

Let us denote by $\eta\left(x^{1 / n}\right) \in \mathbb{K}\left[\left[x^{1 / n}\right]\right]$ a Newton-Puiseux series of $f(x, y)$ with respect to $x$, where $\eta \in \mathbb{K}[[t]]$. We assume that the representation of this Newton-Puiseux power series is primitive (see Definition 2.5). The series $\eta$ is of the form:

$$
\eta=a \cdot t^{m}+\text { higher order terms, }
$$

with $m>0$ and $a \in \mathbb{K}^{*}$. Let us choose an $m$-th root $\tilde{a} \in \mathbb{K}^{*}$ of $a$. Then, we have a unique $m$-th root $t \tilde{\eta}(t) \in \mathbb{K}[[t]]$ of $\eta$ :

$$
\eta(t)=(t \tilde{\eta}(t))^{m},
$$

such that the series $\tilde{\eta}$ has constant term $[\tilde{\eta}]_{0}=\tilde{a} \neq 0$.

Example 4.1. Start from a branch $C$ with Newton-Puiseux series: $\psi=x^{3 / 2}+c \cdot x^{7 / 4}$, where $c \in \mathbb{K}^{*}$. Therefore, we get $a=1, n=4$ and $\psi=\eta\left(x^{1 / 4}\right)$, where $\eta(t)=t^{6}+c \cdot t^{7}=t^{6}(1+c \cdot t)$. This shows that $m=6$ and if $\tilde{a}=1$ then $\tilde{\eta}(t)=(1+c \cdot t)^{1 / 6}$. By (4.20) below we have the expansion $\tilde{\eta}(t)=1+\sum_{k \in \mathbb{N}^{*}}\left(\begin{array}{c}1 / 6 \\ k\end{array}\right) c^{k} \cdot t^{k}$.

Let us come back to the general case.

Denote by $\tilde{\xi}(u) \in \mathbb{K}[[u]]^{*}$ the dual series of $\tilde{\eta}(t)$ (see Definition 3.14). Hence, one has the following equivalence (see (3.1)):

$$
u=t \tilde{\eta}(t) \Leftrightarrow t=u \tilde{\xi}(u)
$$

As $\eta\left(x^{1 / n}\right)$ is a Newton-Puiseux-series of $f(x, y)$ relative to $x$, we have:

$$
f\left(x, \eta\left(x^{1 / n}\right)\right)=0 .
$$


Replacing $x$ by $t^{n}$ and using the equality (4.1), we get:

$$
f\left(t^{n},(t \tilde{\eta}(t))^{m}\right)=0 .
$$

By the equivalence (4.2), we deduce:

$$
f\left((u \tilde{\xi}(u))^{n}, u^{m}\right)=0 .
$$

Consequently, if one defines:

$$
\xi(u)=(u \tilde{\xi}(u))^{n}
$$

(an equation which is analogous to (4.1)), then one sees that:

$$
f\left(\xi\left(y^{1 / m}\right), y\right)=0,
$$

that is, $\xi\left(y^{1 / m}\right)$ is a Newton-Puiseux series of $f(x, y)$ with respect to the variable $y$.

In fact, one has the following proposition:

\section{Proposition 4.2.}

(1) The map

$$
x^{1 / n} \tilde{\eta}\left(x^{1 / n}\right) \longrightarrow y^{1 / m} \tilde{\xi}\left(y^{1 / m}\right)
$$

induced by the duality involution on $\mathbb{K}[[t]]^{*}$, gives a bijection from the set of $m$-th roots of the Newton-Puiseux series of $f(x, y)$ relative to $x$ to the set of $n$-th roots of those relative to $y$.

(2) If $\tilde{f}(t, u):=f\left(t^{n}, u^{m}\right) \in \mathbb{K}[[t, u]]$ then:

- the series of the form $t \tilde{\eta}(t) \in \mathbb{K}[[t]]$ are the Newton-Puiseux series of $\tilde{f}$ relative to $t$.

- the series of the form $u \tilde{\xi}(u) \in \mathbb{K}[[u]]$ are the Newton-Puiseux series of $\tilde{f}$ relative to $u$.

Proof.

(11) The two sets have both $m n$ elements and the given map is injective because the map $\tilde{\eta} \rightarrow \tilde{\xi}$ is an involution. Therefore the given map is bijective.

(2) Equation (4.3) shows that the Newton-Puiseux series of $\tilde{f}$ relative to the variable $t$ are exactly those of the form $t \tilde{\eta}(t)$. The situation is analogous for the series of the form $u \tilde{\xi}(u)$.

The following lemma relates special sequences of essential exponents of the series $\eta(t)$ and $\tilde{\eta}(t)$ on one side, and of the series $\xi(u)$ and $\tilde{\xi}(u)$ on another side:

Lemma 4.3. Denote:

$$
\left\{\begin{array}{l}
\operatorname{ess}(\eta, n)=\left(m, \epsilon_{1}, \ldots, \epsilon_{d}\right), \\
\operatorname{ess}(\xi, m)=\left(n, \epsilon_{1}^{\prime}, \ldots, \epsilon_{d^{\prime}}^{\prime}\right) .
\end{array}\right.
$$

Then:

$$
\left\{\begin{array}{l}
\operatorname{ess}(\tilde{\eta}, \operatorname{gcd}(n, m))=\left(0, \epsilon_{1}-m, \ldots, \epsilon_{d}-m\right) \\
\operatorname{ess}(\tilde{\xi}, \operatorname{gcd}(n, m))=\left(0, \epsilon_{1}^{\prime}-n, \ldots, \epsilon_{d^{\prime}}^{\prime}-n\right) .
\end{array}\right.
$$

Proof. By symmetry, it is enough to treat the case of the series $\tilde{\eta}$. Since $\tilde{\eta} \in \mathbb{K}[[t]]^{*}$, Proposition 3.16 implies that $\operatorname{Irr}(\tilde{\eta})=\operatorname{Irr}\left(\tilde{\eta}^{m}\right)$. Then, by Lemma 3.11 for any integer $p \in \mathbb{N}^{*}$ one has:

$$
\operatorname{ess}(\tilde{\eta}, p)=\operatorname{ess}(\operatorname{Irr}(\tilde{\eta}), p)=\operatorname{ess}\left(\operatorname{Irr}\left(\tilde{\eta}^{m}\right), p\right)=\operatorname{ess}\left(\tilde{\eta}^{m}, p\right) .
$$

Thus, it is enough to prove that $\left(0, \epsilon_{1}-m, \ldots, \epsilon_{d}-m\right)$ is the sequence of essential exponents of $\tilde{\eta}^{m}$ relative to $\operatorname{gcd}(n, m)$. By formula (4.1), we have that $\tilde{\eta}^{m}=t^{-m} \eta$, therefore: $\mathcal{S}\left(\tilde{\eta}^{m}\right)=\mathcal{S}(\eta)-m$. Using Definition 3.6. we see that we have to prove that:

- $\min (\mathcal{S}(\eta)-m)=0$.

- For all $k \in\{1, \ldots, d\}: \epsilon_{k}-m=\min \left((\mathcal{S}(\eta)-m) \backslash \mathbb{Z}\left\{\operatorname{gcd}(n, m), 0, \epsilon_{1}-m, \ldots, \epsilon_{k-1}-m\right\}\right)$.

- $\mathcal{S}(\eta)-m \subset \mathbb{Z}\left\{\operatorname{gcd}(n, m), 0, \epsilon_{1}-m, \ldots, \epsilon_{d}-m\right\}$.

But all these facts are immediate from the definition of the essential exponents $\epsilon_{i}$, because:

$$
\mathbb{Z}\left\{\operatorname{gcd}(n, m), 0, \epsilon_{1}-m, \ldots, \epsilon_{k-1}-m\right\}=\mathbb{Z}\left\{n, m, \epsilon_{1}, \ldots, \epsilon_{k-1}\right\},
$$

for all $1 \leqslant k \leqslant d$, an equality which is immediate to check by double inclusion. 
We are ready to deduce an inversion formula, expressing the sequence of essential exponents of $\xi$ relative to $m$ and the associated coefficients in terms of the sequence of essential exponents of $\eta$ relative to $n$ and their associated coefficients. We chose to inverse also the order of presentation, by starting from any pair of dual series $(\tilde{\eta}, \tilde{\xi})$ and any pair of positive integers $(m, n)$, and by associating to them the series $(\eta, \xi)$ by the formulae (4.1) and (4.5). In this way, we emphasize only univalued maps, in contrast to their reciprocals, which involve taking roots.

Theorem 4.4. Let $\tilde{\eta} \in \mathbb{K}[[t]]^{*}$ and $\tilde{\xi} \in \mathbb{K}[[u]]^{*}$ be dual of each other and $m, n \in \mathbb{N}^{*}$. Let a be the constant term of $\tilde{\eta}$. Denote:

$$
\left\{\begin{array}{l}
\eta(t)=(t \tilde{\eta}(t))^{m} \\
\xi(u)=(u \tilde{\xi}(u))^{n}
\end{array}\right.
$$

and:

$$
\left\{\begin{array}{l}
\operatorname{ess}(\eta, n)=\left(m, \epsilon_{1}, \ldots, \epsilon_{d}\right), \\
\operatorname{ess}(\xi, m)=\left(n, \epsilon_{1}^{\prime}, \ldots, \epsilon_{d^{\prime}}^{\prime}\right) .
\end{array}\right.
$$

Then one has the following inversion formulae for exponents and coefficients:

$$
\begin{gathered}
d^{\prime}=d, \\
\epsilon_{k}^{\prime}+m=\epsilon_{k}+n, \text { for all } k \in\{1, \ldots, d\} \\
{[\xi]_{n}=\tilde{a}^{-n} \quad \text { and } \quad[\xi]_{\epsilon_{k}^{\prime}}=-\frac{n}{m} \tilde{a}^{-n-\epsilon_{k}}[\eta]_{\epsilon_{k}}, \text { for all } k \in\{1, \ldots, d\} .}
\end{gathered}
$$

Proof. The entire series $\tilde{\eta}$ and $\tilde{\xi}$ being dual in the sense of Definition 3.14. Corollary 3.17 shows that they have the same sequences of essential exponents relative to $\operatorname{gcd}(n, m)$. Then, Lemma 4.3 allows us to deduce the desired formulae (4.6) and (4.7) relating the two sequences $\left(\epsilon_{k}\right)_{k}$ and $\left(\epsilon_{k}^{\prime}\right)_{k}$.

Let us pass to the proof of the inversion formula (4.8) for the coefficients.

Equation (4.5) implies that $\xi=u^{n}(\tilde{\xi})^{n}$. Therefore:

$$
[\xi]_{\epsilon_{k}^{\prime}}=\left[(\tilde{\xi})^{n}\right]_{\epsilon_{k}^{\prime}-n} .
$$

Combining Proposition 3.16 and Lemma 3.10, we get:

$$
\left[(\tilde{\xi})^{n}\right]_{\epsilon_{k}^{\prime}-n}=n[\tilde{\xi}]_{0}^{n-1}[\tilde{\xi}]_{\epsilon_{k}^{\prime}-n}=n \tilde{a}^{-n+1}[\tilde{\xi}]_{\epsilon_{k}^{\prime}-n} .
$$

The same proposition, combined with the equivalent form $\epsilon_{k}^{\prime}-n=\epsilon_{k}-m$ of the equality (4.7), implies that:

$$
[\tilde{\xi}]_{\epsilon_{k}^{\prime}-n}=-[\tilde{\eta}]_{0}^{-\epsilon_{k}+m-2}[\tilde{\eta}]_{\epsilon_{k}-m}=-\tilde{a}^{-\epsilon_{k}+m-2}[\tilde{\eta}]_{\epsilon_{k}-m}
$$

Combining the equalities (4.9), (4.10) and (4.11), we obtain:

$$
[\xi]_{\epsilon_{k}^{\prime}}=-n \tilde{a}^{-\epsilon_{k}+m-n-1}[\tilde{\eta}]_{\epsilon_{k}-m} .
$$

Now, from the analogues of equations (4.9) and (4.10) for $\tilde{\eta}$, we get:

$$
[\tilde{\eta}]_{\epsilon_{k}-m}=\frac{1}{m}[\tilde{\eta}]_{0}^{-m+1}\left[\tilde{\eta}^{m}\right]_{\epsilon_{k}-m}=\frac{1}{m}[\tilde{\eta}]_{0}^{-m+1}[\eta]_{\epsilon_{k}}=\frac{1}{m} \tilde{a}^{-m+1}[\eta]_{\epsilon_{k}} .
$$

Combining formulae (4.12) and (4.13), we deduce the inversion formula for the coefficients $[\xi]_{\epsilon_{k}^{\prime}}$, for $k \in\{1, \ldots, d\}$.

Dividing by $n$ all the terms of the sequence $\left(m, \epsilon_{1}, \ldots, \epsilon_{d}\right)$, one gets the sequence of essential exponents of $\eta$ relative to 1 (see Remark 3.7). Similarly, dividing by $m$ all the terms of the sequence $\left(n, \epsilon_{1}^{\prime}, \ldots, \epsilon_{d}^{\prime}\right)$, one gets the sequence of essential exponents of $\xi$ relative to 1 . Theorem 4.4 translates therefore in the following inversion formula for the Newton-Puiseux series of $f(x, y)$ relative to $x$ and to $y$, which is the theorem of Halphen-Stolz presented in the introduction: 


\section{Corollary 4.5 (The Halphen-Stolz inversion theorem).}

Let $\eta\left(x^{1 / n}\right)$ and $\xi\left(y^{1 / m}\right)$ be Newton-Puiseux series of an irreducible formal power series $f(x, y) \in \mathbb{K}[[x, y]]$ relative to $x$ and $y$ respectively. As before, we assume that $\eta(t)=(t \tilde{\eta}(t))^{m}$ and $\xi(u)=(u \tilde{\xi}(u))^{n}$, where $\tilde{\eta}(t), \tilde{\xi}(u)$ are dual series and $[\tilde{\eta}]_{0}=\tilde{a}$. Denote:

$$
\left\{\begin{array}{l}
\operatorname{ess}\left(\eta\left(x^{1 / n}\right), 1\right)=\left(m / n, e_{1}, \ldots, e_{d}\right) \\
\operatorname{ess}\left(\xi\left(y^{1 / m}\right), 1\right)=\left(n / m, e_{1}^{\prime}, \ldots, e_{d^{\prime}}^{\prime}\right) .
\end{array}\right.
$$

Then one has the following inversion formulae for exponents and coefficients:

$$
\begin{gathered}
d^{\prime}=d . \\
m\left(1+e_{k}^{\prime}\right)=n\left(1+e_{k}\right) \text { for all } k \in\{1, \ldots, d\} . \\
{\left[\xi\left(y^{1 / m}\right)\right]_{n / m}=\tilde{a}^{-n} \text { and }\left[\xi\left(y^{1 / m}\right)\right]_{e_{k}^{\prime}}=-\frac{n}{m} \tilde{a}^{-\left(1+e_{k}\right) n}\left[\eta\left(x^{1 / n}\right)\right]_{e_{k}} \text { for all } k \in\{1, \ldots, d\} .}
\end{gathered}
$$

In the case in which $\tilde{a}=1$, the inversion formula for the coefficients stated in Corollary 4.5 may be written in a more symmetric way, easier to remember:

Corollary 4.6. Assume moreover that the constant coefficient $\tilde{a}$ of $\tilde{\eta}$ is equal to 1 . Then:

$$
\left[\xi\left(y^{1 / m}\right)\right]_{n / m}=1=\left[\eta\left(x^{1 / n}\right)\right]_{m / n} \quad \text { and } \quad m\left[\xi\left(y^{1 / m}\right)\right]_{e_{k}^{\prime}}+n\left[\eta\left(x^{1 / n}\right)\right]_{e_{k}}=0 \text { for all } k \in\{1, \ldots, d\} .
$$

Summary of the previous arguments. In order to understand better the line of reasoning we followed till now, the reader may find helpful the following flow-chart, in which $f(x, y) \in \mathbb{K}[[x, y]]$ is irreducible:

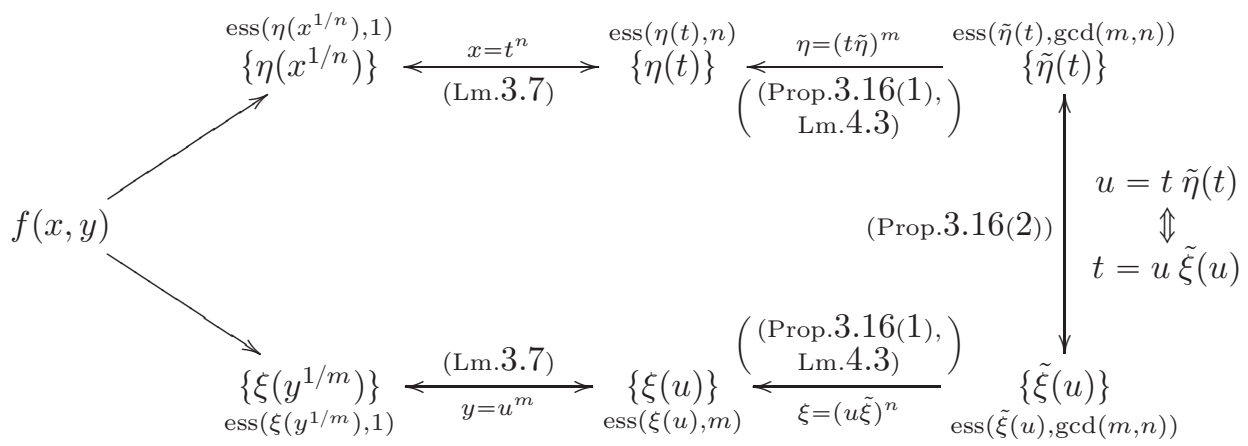

Let us explain this diagram:

- From the irreducible series $f(x, y) \in \mathbb{K}[[x, y]]$, one gets symmetrically two sets of Newton-Puiseux series $\left\{\eta\left(x^{1 / n}\right)\right\}$ and $\left\{\xi\left(y^{1 / m}\right)\right\}$. The first one has $n$ and the second one $m$ elements.

- Follow now two analogous sequences of transformations of those sets, indicated in the diagram horizontally. We describe them only for the upper line of the diagram.

- The change of variables $x=t^{n}$, indicated above the corresponding doubly-arrowed horizontal segment, puts the set $\left\{\eta\left(x^{1 / n}\right)\right\}$ in bijection with the set of entire series $\{\eta(t)\}$.

- Lemma 3.7 mentioned below the same arrow, allows to pass from $\operatorname{ess}\left(\eta\left(x^{1 / n}\right), 1\right)$ to $\operatorname{ess}(\eta(t), n)$. The corresponding coefficients are unchanged.

- One extracts in all possible ways the $m$-th roots of the series $\eta(t)$. Then one divides the result by $t$, arriving at a set $\{\tilde{\eta}(t)\}$ with $m n$ elements. The composition of the two operations is expressed by the formula $\eta=(t \tilde{\eta})^{m}$, written above the corresponding arrow.

- Combining Proposition 3.16 (1) with Lemma 4.3. one passes from the sequence ess $(\eta(t), n)$ to $\operatorname{ess}(\tilde{\eta}(t), \operatorname{gcd}(m, n))$ and one relates also the corresponding coefficients.

- There is a canonical bijection between the two sets $\{\tilde{\eta}(t)\}$ and $\{\tilde{\xi}(u)\}$, indicated by the left vertical double-arrowed segment. This bijection associates two series $\tilde{\eta}(t)$ and $\tilde{\xi}(u)$ whenever $\tilde{\eta}(t)$ and $\tilde{\xi}(u)$ are dual of each other, which may be expressed by the two equivalent equalities marked at the right of the vertical segment. 
- Proposition 3.16 (2), indicated to the left of the same segment, shows that the two sequences are equal, and allows to relate the corresponding coefficients. Note that this proposition allows in fact to relate the coefficients corresponding to all the irreducible exponents of the two dual series, not only those which are essential relative to $\operatorname{gcd}(m, n)$. This is understandable if one thinks that, reading now the diagram from right to left, one may start from any pair $(\tilde{\eta}(t), \tilde{\xi}(u))$ of dual series and only afterwards choose the pair of positive integers $(m, n)$, independently of the choice of the two dual series. One arrives then at the series $f(x, y)$ by taking either the minimal polynomial of $\eta\left(x^{1 / n}\right)$ or that of $\xi\left(y^{1 / m}\right)$, and multiplying it with an invertible element of the ring $\mathbb{K}[[x, y]]$.

Remark 4.7. Using Lemma 3.13, the Halphen-Stolz inversion theorem (Corollary 4.5) may also be expressed in terms of the characteristic exponents of $\eta$ and $\xi$. That lemma shows that the sequences of characteristic exponents of $\eta$ and of $\xi$ do not have necessarily the same lengths, which has as consequence the fact that the elements of the two series which are related have not necessarily the same position in both sequences. For this reason, it is easier to express the inversion formulae as we have done in Theorem 4.4 and in Corollary 4.5, in terms of the essential exponents.

Remark 4.8. The part of Corollary 4.5 concerning the exponents is usually expressed nowadays in terms of the characteristic exponents and is sometimes attributed to Abhyankar's paper [2] of 1967 or to Zariski's paper [40] of 1968. In fact, it was already stated precisely in terms of the sequences of essential exponents relative to 1 (called there "exposants caractéristiques" from their second term on) by Halphen [20, page 91] in 1876. But Halphen stated also the previous formulae (of course, with different notations) for the inversion of the corresponding coefficients. He did not prove those formulae, and as far as we know, the unique proof was provided by Stolz [36, page 133] in 1879. We searched new proofs because we were not fully convinced by Stolz' arguments and because we wanted to extend the theorem to higher dimensions.

\subsection{The second proof of the Halphen-Stolz theorem.}

Let us pass now to our second proof of Theorem 4.4. Corollary 4.5 concerns only the terms of the two Newton-Puiseux series whose exponents are essential relative to 1 . We explain now a way to get formulae for all the coefficients of $\xi$ as rational fractions of those of $\eta$.

We recall first a form of Lagrange's inversion formula which, given two reciprocal entire series $X$ and $Y$, allows to express the coefficients of the integral powers of $X$ in terms of those of $Y$. Several proofs of it may be found in [35, Theorem 5.4.2], and historical explanations in [35, pages 67-68]. Let us mention only that the founding result for this kind of formulae was stated by Lagrange in [23, Par. 16].

Theorem 4.9 (Lagrange inversion formula). Let $X(u) \in u \mathbb{K}[[u]]^{*}$ and $Y(t) \in t \mathbb{K}[[t]]^{*}$ be two reciprocal series. For any $p, q \in \mathbb{Z}$, one has:

$$
p \cdot\left[X(u)^{q}\right]_{p}=q \cdot\left[Y(t)^{-p}\right]_{-q} .
$$

Note that the lifting to a negative integral power produces a meromorphic series which has a finite number of terms with negative exponents.

Let us apply Theorem 4.9 in our context.

Recall that $\tilde{a} \in \mathbb{K}^{*}$ is the constant term of $\tilde{\eta}$, hence by formula [4.1], we may write:

$$
\eta(t)=\tilde{a}^{m} t^{m}\left(1+\sum_{k>m} c_{k} t^{k-m}\right) .
$$

Therefore:

$$
\tilde{\eta}(t)=\tilde{a}\left(1+\sum_{k>m} c_{k} t^{k-m}\right)^{1 / m},
$$


in which the right-hand-side can be computed using the generalized binomial expansion:

$$
(1+x)^{r}:=1+\sum_{k \in \mathbb{N}^{*}}\left(\begin{array}{l}
r \\
k
\end{array}\right) x^{k}, \forall r \in \mathbb{R}
$$

where:

$$
\left(\begin{array}{l}
r \\
k
\end{array}\right)=\frac{r(r-1) \cdots(r-k+1)}{k !}, \text { for all } r \in \mathbb{R} .
$$

Using formula (4.19), one gets the following consequence of Theorem 4.9, which allows to compute the coefficients of the Newton-Puiseux series $\xi$ of the irreducible power series $f(x, y) \in \mathbb{K}[[x, y]]$ relative to $y$ in terms of those $\eta$ relative to $x$. Note that one gets rational fractions whose numerators are polynomials with rational coefficients in the coefficients $c_{k}$ and whose denominators are positive integral powers of $\tilde{a}$ :

Proposition 4.10. Assume that:

$$
\eta\left(x^{1 / n}\right)=\tilde{a}^{m} x^{m / n}\left(1+\sum_{k>m} c_{k} x^{\frac{k-m}{n}}\right) .
$$

Then one has the following formula for the coefficients $[\xi]_{\frac{q}{m}}$ of the corresponding Newton-Puiseux series $\xi\left(y^{1 / m}\right) \in \mathbb{K}\left[\left[y^{1 / m}\right]\right]$, for all integer $q \geqslant n$ :

$$
[\xi]_{\frac{q}{m}}=\frac{n}{q} \tilde{a}^{-q}\left[1+\sum_{i \geqslant 1}\left(\begin{array}{c}
-q / m \\
i
\end{array}\right)\left(\sum_{s>m} c_{s} x^{\frac{s-m}{n}}\right)^{i}\right]_{-1+\frac{q}{n}} .
$$

Proof. By Theorem 4.9 applied after replacing the pair $(p, q)$ by $(q, n)$, we have:

$$
q \cdot\left[u^{n} \tilde{\xi}(u)^{n}\right]_{q}=n \cdot\left[t^{-q} \tilde{\eta}(t)^{-q}\right]_{-n} .
$$

We get:

$$
q \cdot\left[u^{n} \tilde{\xi}(u)^{n}\right]_{q} \stackrel{4.19}{=} n \tilde{a}^{-q}\left[\left(t^{-q}\left(1+\sum_{s>m} c_{s} t^{s-m}\right)^{-q / m}\right)\right]_{-n},
$$

and then by (4.20):

$$
q \cdot[\xi(u)]_{q}=q \cdot\left[u^{n} \tilde{\xi}(u)^{n}\right]_{q}=n \tilde{a}^{-q}\left[\left(1+\sum_{i \geqslant 1}\left(\begin{array}{c}
-q / m \\
i
\end{array}\right)\left(\sum_{s>m} c_{s} t^{s-m}\right)^{i}\right)\right]_{q-n} .
$$

It is enough now to divide by $q$, to replace $u$ by $y^{1 / m}$ and $t$ by $x^{1 / n}$ in order to get the desired formula.

As a corollary, we obtain:

Second proof of Theorem 4.4. Recall the notation ess $(\eta, n)=\left(m, \epsilon_{1}, \ldots, \epsilon_{d}\right)$. We set then:

$$
\hat{\epsilon}_{0}=n, \quad \hat{\epsilon}_{1}=\epsilon_{1}-m+n, \quad \ldots, \quad \hat{\epsilon}_{d}=\epsilon_{d}-m+n .
$$

We prove first by induction on the integer $q \geqslant n$ that if

$$
\hat{\epsilon}_{k} \leqslant q<\hat{\epsilon}_{k+1} \text { for some } k \in\{0, \ldots, d\},
$$

then the terms of the sequence $\operatorname{ess}(\xi, m)$ which are lower than or equal to $q$ are precisely $\hat{\epsilon}_{0}=n, \hat{\epsilon}_{1}, \ldots, \hat{\epsilon}_{k}$. Here the case $k=d$ in (4.24) means simply that $\hat{\epsilon}_{d} \leqslant q$.

If $q=n$, we get from (4.23) that $[\xi]_{n}=\tilde{a}^{-n} \neq 0$ is the dominant term of the series $\xi$, hence the assertion (4.24) holds by Definition 3.12. Assume that (4.24) holds for some $q>n$. We distinguish two cases:

- Assume that $\hat{\epsilon}_{k}<q$, so $\hat{\epsilon}_{k} \leqslant q-1$. Then, by the induction hypothesis applied to $q-1$, we have that the terms of the sequence $\operatorname{ess}(\xi, m)$ which are lower than or equal to $q-1$ are precisely $\hat{\epsilon}_{0}=n, \hat{\epsilon}_{1}, \ldots, \hat{\epsilon}_{k}$. If $[\xi(u)]_{q}=0$, then there is nothing to prove. Assume that $[\xi(u)]_{q} \neq 0$. Since $q-n<\epsilon_{k+1}-m$ by 
(4.24), the exponent of a term appearing in the polynomial $\left(\sum_{m<s<\epsilon_{k+1}} c_{s} t^{s-m}\right)^{i}$ must belong to the group $\mathbb{Z}\left\{n, m, \epsilon_{1}, \ldots, \epsilon_{k}\right\}$, by the definition of the essential exponents $\operatorname{ess}(\eta, n)$. We deduce from this and the right hand side of the equality (4.23) that $q$ must belong to the subgroup $\mathbb{Z}\left\{n, m, \epsilon_{1}, \ldots, \epsilon_{k}\right\}$. Since by definition we have the equality:

$$
\mathbb{Z}\left\{n, m, \epsilon_{1}, \ldots, \epsilon_{k}\right\}=\mathbb{Z}\left\{n, m, \hat{\epsilon}_{1}, \ldots, \hat{\epsilon}_{k}\right\}
$$

$q$ cannot be an essential exponent of $\xi$ with respect to $n$ (see Definition 3.12 ).

- Assume that $\hat{\epsilon}_{k}=q$. Then, by the induction hypothesis applied to $q-1$, we have that the terms of the sequence $\operatorname{ess}(\xi, m)$ which are lower or equal to $q-1$ are precisely $\hat{\epsilon}_{0}=n, \hat{\epsilon}_{1}, \ldots, \hat{\epsilon}_{k-1}$. We have to prove that the coefficient $[\xi(u)]_{q}$ does not vanish. Notice that there is a term with exponent equal to $q-n$ appearing in the polynomial $\left(\sum_{m<s \leqslant \epsilon_{k}} c_{s} t^{s-m}\right)^{i}$ if and only if $i=1$ and then this term is equal to $c_{\epsilon_{k}} t^{q-n}$. Indeed, arguing as in the previous case, we see that any other term would provide an expansion of $\epsilon_{k}=q-n+m$ in the group $\mathbb{Z}\left\{n, m, \epsilon_{1}, \ldots, \epsilon_{k-1}\right\}$, contradicting the definition of the essential exponent $\epsilon_{k}$. Notice that $c_{\epsilon_{k}}=\tilde{a}^{-m}[\eta]_{\epsilon_{k}}$, by (4.18). It follows from (4.23) that:

$$
q \cdot[\xi]_{q}=n \tilde{a}^{-\epsilon_{k}+m-n}\left(\frac{-q}{m}\right) \tilde{a}^{-m}[\eta]_{\epsilon_{k}}=-q \frac{n}{m} \tilde{a}^{-\epsilon_{k}-n}[\eta]_{\epsilon_{k}},
$$

thus $[\xi]_{q}=-\frac{n}{m} \tilde{a}^{-\epsilon_{k}-n}[\eta]_{\epsilon_{k}}$ is nonzero. This finishes the proof of the assertion.

Theorem 4.4 is proved, since we have also proved the inversion formula (4.8) for the coefficients.

Example 4.11. Let us consider again the Newton-Puiseux series $\eta\left(x^{1 / 4}\right)$ of Example 4.1. Denote by $\xi\left(y^{1 / 6}\right)$ a Newton-Puiseux series corresponding by inversion to $\eta$, through the bijection described in Proposition 4.2, Applying Proposition 4.10, we get:

$$
\left[\xi\left(y^{1 / 6}\right)\right]_{p / 6}=\frac{4}{p}\left[1+\sum_{i \geqslant 1}\left(\begin{array}{c}
-p / 6 \\
i
\end{array}\right)\left(c x^{\frac{1}{4}}\right)^{i}\right]_{-1+\frac{p}{4}}=\frac{4}{p}\left(\begin{array}{c}
-p / 6 \\
p-4
\end{array}\right) c^{p-4} .
$$

That is:

$$
\xi\left(y^{1 / 6}\right)=\sum_{p \geqslant 4} \frac{4}{p}\left(\begin{array}{c}
-p / 6 \\
p-4
\end{array}\right) c^{p-4} y^{p / 6} .
$$

The first two exponents in the support $\mathcal{S}\left(\xi\left(y^{1 / 6}\right)\right)$ are therefore $4 / 6=2 / 3$ and $5 / 6$, which shows that they constitute the characteristic sequence of $\xi$. The corresponding terms of $\xi$ are, according to the previous formula: $1 \cdot y^{2 / 3},\left(-\frac{2}{3} c\right) \cdot y^{5 / 6}$. One may verify then immediately the correcteness of the formulae stated in the Halphen-Stolz inversion theorem (Corollary 4.5).

Remark 4.12. In order to compute recursively the coefficients of $\xi(y)$ starting from those of $\eta(x)$, one could also use the method explained by Borodzik [4].

Remark 4.13. We believe that one can use Abhyankar's [3, First Inversion Theorem, page 111] in order to obtain a third proof of the Halphen-Stolz inversion theorem. The approach of that paper seems to be similar in spirit to our first approach.

\section{Generalization to An ARbitrary number of variables}

In this section we generalize our first proof of the Halphen-Stolz theorem to an arbitrary number of variables. We formulate the needed generalizations of the definitions and propositions used in that proof. We only sketch their proofs, insisting in the differences with respect to the one-variable case. Finally, we explain how our result generalizes Lipman's inversion theorem for the characteristic exponents of quasi-ordinary branches.

Throughout the section, we consider a fixed number $h \in \mathbb{N}^{*}$ and we work with the $\mathbb{Q}$-vector space $\mathbb{Q}^{h}$ and various free subgroups of it of rank $h$, which we will call briefly lattices of $\mathbb{Q}^{h}$. We denote by $\left(\nu_{1}, \nu_{2}, \ldots, \nu_{h}\right)$ the canonical basis of $\mathbb{Q}^{h}$. 


\subsection{Irreducible exponents of subsets of $\mathbb{Q}^{h}$ with bounded denominators.}

The notions of set with bounded denominators (Definition 3.1) and of its irreducible elements (Definition 3.2) extend immediately from subsets of $\mathbb{Q}_{+}$to subsets of $\mathbb{Q}_{+}^{h}$. If $E$ is such a set, it generates again a semigroup $\mathbb{N}^{*} E \subset \mathbb{Q}_{+}^{h}$ and a group $\mathbb{Z} E \subset \mathbb{Q}^{h}$.

Lemma 3.5 (1) remains true in this setting:

Lemma 5.1. If $E \subset \mathbb{Q}_{+}^{h}$ is a set with bounded denominators, then $\operatorname{Irr}(E)=\operatorname{Irr}\left(\mathbb{N}^{*} E\right)$ and this set is the minimal generating set of the semigroup $\mathbb{N}^{*} E$, relative to the inclusion partial order between its generating sets.

Notice that point (2) of Lemma 3.5 is not necessarily true for $h \geqslant 2$, as shown by the following standard example:

Example 5.2. Take $E=\left(\mathbb{N}^{*}\right)^{2}$. Then $\operatorname{Irr}(E)=\left(\mathbb{N}^{*} \times\{1\}\right) \cup\left(\{1\} \times \mathbb{N}^{*}\right)$. Therefore $\operatorname{Irr}(E)$ is infinite.

When $h \geqslant 2$, we will need also to use special order relations on the group $\left(\mathbb{Q}^{h},+\right)$ :

Definition 5.3. An additive order on $\mathbb{Q}^{h}$ is a partial order relation $\leq$ on $\mathbb{Q}^{h}$ satisfying:

(1) $\leq$ is a total order;

(2) if $\alpha, \beta, \gamma \in \mathbb{Z}^{h}$ and $\alpha \leq \beta$, then $\alpha+\gamma \leq \beta+\gamma$.

The additive order $\leq$ dominates a set $\theta \subset \mathbb{Q}^{h}$ if any non empty subset of $\theta$ with bounded denominators has a minimum relative to $\leq$.

Remark 5.4. If $\leq$ is an additive order of $\mathbb{Q}^{h}$, then there exist an integer $s \in[1, h]$, linear forms $u_{1}, \ldots, u_{s} \in\left(\mathbb{R}^{h}\right)^{*}$, and an increasing injective group morphism:

$$
\left(\mathbb{Q}^{h}, \leq\right) \rightarrow\left(\mathbb{R}^{h}, \leqslant_{\text {lex }}\right), \quad v \rightarrow\left(u_{1}(v), \ldots, u_{s}(v)\right),
$$

where $\leqslant_{\text {lex }}$ denotes the lexicographical order (see [33, Theorem 2.5]). The lexicographical order is additive and dominates $\mathbb{Z}_{+}^{h}$. More generally, if $\leq$ dominates $\mathbb{Z}_{+}^{h}$, then $\leq$ defines a well-order on $\mathbb{Z}_{+}^{h}$, hence Definition 5.3 is a generalization of the notion of term order explained in [8, Chap. 2.2].

Definition 5.3 allows to generalize the notion of sequence of essential elements relative to an integer $p$ (Definition 3.6) in the following way:

Definition 5.5. Let us consider a set $E \subset \mathbb{Q}_{+}^{h}$ with bounded denominators. Let $M$ be a lattice of $\left(\mathbb{Q}^{h},+\right)$ and $\leq$ be an additive order on $\mathbb{Q}^{h}$ dominating its subset $\mathbb{Q}_{+}^{h}$. Then the sequence $\operatorname{ess}(E, M, \leq):=\left(\operatorname{ess}(E, M, \leq)_{l}\right)_{l}$ of essential elements of $E$ relative to $M$ is defined inductively by:

- $\operatorname{ess}(E, M, \leq)_{0}:=\min E$.

- If $l \geqslant 1$, then the term $\operatorname{ess}(E, M, \leq)_{l}$ is defined if and only if $E$ is not included in the group $M+\mathbb{Z}\left\{\operatorname{ess}(E, M, \leq)_{0}, \ldots, \operatorname{ess}(E, M, \leq)_{l-1}\right\}$. In this case:

$$
\operatorname{ess}(E, M, \preceq)_{l}:=\min \left(E \backslash\left(M+\mathbb{Z}\left\{\operatorname{ess}(E, M, \preceq)_{0}, \ldots, \operatorname{ess}(E, M, \preceq)_{l-1}\right\}\right)\right) .
$$

One gets Definition 3.6 by taking $h=1, M=p \mathbb{Z}$ and $\leq$ to be the unique additive order on $(\mathbb{Q},+)$ which dominates $\mathbb{Q}_{+}$, that is, the usual order. Indeed, then the sequence ess $(E, p \mathbb{Z}, \leq)$ defined according to Definition $[5.5$ is precisely the sequence ess $(E, p)$ defined according to Definition [3.6

Lemma 3.8 about the finiteness of the sequences ess $(E, M)$ holds also in our larger context:

Lemma 5.6. Assume that the subset $E \subset \mathbb{Q}_{+}^{h}$ has bounded denominators, that $M$ is a lattice of $\mathbb{Q}^{h}$ and that $\leq$ is an additive order dominating $\mathbb{Q}_{+}^{h}$. Then the sequence of essential exponents $\operatorname{ess}(E, M, \leq)$ of $E$ relative to $M$ is finite.

Proof. For every integer $l \geqslant 0$ for which $\operatorname{ess}(E, M, \leq)_{l}$ is defined, let us denote by $M_{l}$ the abelian group $M+\mathbb{Z}\left\{\operatorname{ess}(E, M, \leq)_{0}, \ldots, \operatorname{ess}(E, M, \leq)_{l}\right\}$. Since $\left(M_{l}\right)_{l}$ is an increasing sequence of abelian groups, the union $\bigcup_{l} M_{l}$ is also an abelian group. The hypothesis that $E$ has bounded denominators implies that this group $\bigcup_{l} M_{l}$ is a lattice of $\mathbb{Q}^{h}$. Any ascending chain of subgroups of a free abelian group of finite rank being stationary, the sequence $\left(M_{l}\right)_{l}$ must be finite. Therefore, the sequence ess $(E, M, \leq)$ is also finite. 
If $q \in \mathrm{GL}(h, \mathbb{Q})$ and if $\leq$ is a additive order on $\mathbb{Q}^{h}$, we denote by $\leq_{q}$ the additive order defined by:

$$
\alpha \unlhd_{q} \beta \Leftrightarrow q(\alpha) \leq q(\beta) .
$$

By using this notion, Lemma 3.7 extends immediately into:

Lemma 5.7. Assume that $E \subset \mathbb{Q}_{+}^{h}$ has bounded denominators and that $M$ is a lattice of $\mathbb{Q}^{h}$. Take $q \in G L(h, \mathbb{Q})$ such that $q\left(\mathbb{Q}_{+}^{h}\right) \subset \mathbb{Q}_{+}^{h}$ and let $\leq$ be a additive order dominating $\mathbb{Q}_{+}^{h}$. Then:

$$
q\left(\operatorname{ess}\left(E, M, \leq_{q}\right)\right)=\operatorname{ess}(q(E), q(M), \leq) .
$$

Lemmas 3.10 and 3.11 also extend immediately to our more general context:

Lemma 5.8. The essential elements of a set $E \subset \mathbb{Q}_{+}^{h}$ with bounded denominators relative to any lattice $M$ of $\mathbb{Q}^{h}$ and an additive order $\leq$ dominating $\mathbb{Q}_{+}^{h}$ are irreducible elements of $E$.

Lemma 5.9. Let $E \subset \mathbb{Q}_{+}^{h}$ be a set with bounded denominators, $M$ be a lattice of $\mathbb{Q}^{h}$ and $\leq$ be an additive order dominating $\mathbb{Q}_{+}^{h}$. Then we have the following equality of essential sequences:

$$
\operatorname{ess}(E, M, \preceq)=\operatorname{ess}(\operatorname{Irr}(E), M, \preceq) .
$$

\subsection{On the notions of dual and reciprocal series in several variables.}

Consider now the ring $\mathbb{K}\left[\left[t_{1}, t_{2}, \ldots, t_{h}\right]\right]$, and its subset:

$$
\mathbb{K}\left[\left[t_{1}, t_{2}, \ldots, t_{h}\right]\right]^{*}=\left\{\phi \in \mathbb{K}\left[\left[t_{1}, t_{2}, \ldots, t_{h}\right]\right]: \phi(0, \ldots, 0) \neq 0\right\}
$$

consisting of the series with non-zero constant term. It is the group of multiplicatively invertible elements of the ring $\mathbb{K}\left[\left[t_{1}, t_{2}, \ldots, t_{h}\right]\right]$.

If $\phi \in \mathbb{K}\left[\left[t_{1}, t_{2}, \ldots, t_{h}\right]\right]^{*}$ has constant term $\alpha \neq 0$, then the map:

$$
\begin{aligned}
\mathbb{K}\left[\left[t_{1}, t_{2}, \ldots, t_{h}\right]\right] & \longrightarrow \mathbb{K}\left[\left[t_{1}, t_{2}, \ldots, t_{h}\right]\right] \\
\left(t_{1}, t_{2}, \ldots, t_{h}\right) & \longrightarrow\left(t_{1} \phi\left(t_{1}, t_{2}, \ldots, t_{h}\right), t_{2}, \ldots, t_{h}\right)
\end{aligned}
$$

is invertible for composition, as its linearization $\left(t_{1}, t_{2}, \ldots, t_{h}\right) \rightarrow\left(\alpha t_{1}, t_{2}, \ldots, t_{h}\right)$ is invertible in $G L(h, \mathbb{K})$. One has the following generalization of the duality of series in $\mathbb{K}[[t]]^{*}$, introduced in Definition 3.14

Definition 5.10. If $\phi \in \mathbb{K}\left[\left[t_{1}, t_{2}, \ldots, t_{h}\right]\right]^{*}$, then its dual relative to the first variable is the unique entire series $\check{\phi} \in \mathbb{K}\left[\left[u_{1}, t_{2}, \ldots, t_{h},\right]\right]^{*}$ such that the following maps are reciprocal:

$$
\left(u_{1}, t_{2}, \ldots, t_{h}\right) \rightarrow\left(u_{1} \breve{\phi}\left(u_{1}, t_{2}, \ldots, t_{h}\right), t_{2}, \ldots, t_{h}\right), \quad\left(t_{1}, t_{2}, \ldots, t_{h}\right) \rightarrow\left(t_{1} \phi\left(t_{1}, t_{2}, \ldots, t_{h}\right), t_{2}, \ldots, t_{h}\right) .
$$

Remark 5.11. Note that the previous definition depends in an essential way on the choice of the first variable $t_{1}$, but that it is symmetric in the other variables. If $\phi \in \mathbb{K}\left[\left[t_{1}, t_{2}, \ldots, t_{h}\right]\right]^{*}$, then setting $u_{1}=$ $t_{1} \phi\left(t_{1}, t_{2}, \ldots, t_{h}\right)$ defines a change of variables in the ring $\mathbb{K}\left[\left[t_{1}, t_{2}, \ldots, t_{h}\right]\right]$. Notice that $\mathbb{K}\left[\left[t_{1}, t_{2}, \ldots, t_{h}\right]\right]=$ $\mathbb{K}\left[\left[u_{1}, t_{2}, \ldots, t_{h}\right]\right]$ and by Definition 5.10 one has the equivalence:

$$
u_{1}=t_{1} \phi\left(t_{1}, t_{2}, \ldots, t_{h}\right) \Leftrightarrow t_{1}=u_{1} \breve{\phi}\left(u_{1}, t_{2}, \ldots, t_{h}\right) .
$$

The following proposition generalizes Proposition 3.16 to the case of an arbitrary number of variables:

Proposition 5.12. Let $\phi \in \mathbb{K}\left[\left[t_{1}, \ldots, t_{h}\right]\right]^{*}$ and $N \in \mathbb{N}^{*}$. Then:

(1) $\operatorname{Irr}\left(\phi^{N}\right)=\operatorname{Irr}(\phi)$. Moreover $\left[\phi^{N}\right]_{0}=[\phi]_{0}^{N}$ and $\left[\phi^{N}\right]_{r}=N[\phi]_{0}^{N-1}[\phi]_{r}$, for all $r \in \operatorname{Irr}(\phi) \backslash\{0\}$.

(2) $\operatorname{Irr}(\breve{\phi})=\operatorname{Irr}(\phi)$. Moreover $[\check{\phi}]_{0}=[\phi]_{0}^{-1}$ and $[\check{\phi}]_{r}=-[\phi]_{0}^{-r_{1}-2}[\phi]_{r}$, for all $r \in \operatorname{Irr}(\phi) \backslash\{0\}$.

Proof. In what follows, if $k=\left(k_{1}, \ldots, k_{h}\right) \in \mathbb{N}^{h}$, we will write simply:

$$
t^{k}:=t_{1}^{k_{1}} \cdots t_{h}^{k_{h}} .
$$

One has the following analogue of equation (3.2):

$$
\phi(t)=\sum_{j \in \mathcal{S}(\phi)}[\phi]_{j} t^{j} .
$$

The hypothesis $\phi \in \mathbb{K}\left[\left[t_{1}, \ldots, t_{h}\right]\right]^{*}$ translates into $0 \in \mathcal{S}(\phi)$, that is, $[\phi]_{0} \neq 0$. 
(11) Consider first the case of $\phi^{N}$. By equation (5.3), we get the exact analogue of the expansion (3.3):

$$
\phi^{N}(t)=\sum_{j_{1}, \ldots, j_{N} \in \mathcal{S}(\phi)}[\phi]_{j_{1}} \cdots[\phi]_{j_{N}} t^{j_{1}+\cdots+j_{N}} .
$$

Then the proof is identical to that of the one-variable case.

(2) Consider now the case of $\breve{\phi}$. Write the analogue of the expansion (5.3) for the series $\breve{\phi}$ :

$$
\breve{\phi}(u)=\sum_{k \in \mathcal{S}(\breve{\phi})}[\check{\phi}]_{k} u^{k}
$$

where:

$$
u^{k}:=u_{1}^{k_{1}} t_{2}^{k_{2}} \cdots t_{h}^{k_{h}} \text { for all } k=\left(k_{1}, \ldots, k_{h}\right) \in \mathbb{N}^{h} .
$$

By Definition 5.10, if $\breve{\phi}\left(u_{1}, t_{2}, \ldots, t_{h}\right)$ is the dual with respect to $t_{1}$ of the series $\phi\left(t_{1}, t_{2}, \ldots, t_{h}\right)$, then one has the identity:

$$
t_{1}=\left(t_{1} \phi\left(t_{1}, t_{2}, \ldots, t_{h}\right)\right) \cdot \check{\phi}\left(t_{1} \phi\left(t_{1}, \ldots, t_{h}\right), t_{2}, \ldots, t_{h}\right)
$$

which, after division by $t_{1}$ and combination with the expansion (5.5), gives:

$$
1=\sum_{k \in \mathcal{S}(\breve{\phi})}[\check{\phi}]_{k} t^{k} \phi(t)^{k_{1}+1} \text {. }
$$

Expand now the powers $\phi(t)^{k_{1}+1}$ using equation (5.4). We get:

$$
1=\sum_{\substack{k \in \mathcal{S}(\check{\phi}) \\ j_{1}, \ldots, j_{k_{1}+1} \in \mathcal{S}(\phi)}}[\check{\phi}]_{k}[\phi]_{j_{1}} \cdots[\phi]_{j_{k_{1}+1}} t^{k+j_{1}+\cdots+j_{k_{1}+1}} .
$$

Therefore:

$$
\begin{aligned}
& \sum_{k \in \mathcal{S}(\check{\phi})}[\check{\phi}]_{k}[\phi]_{j_{1}} \cdots[\phi]_{j_{k_{1}+1}}=0 \text {, for all } p \in \mathbb{N}^{h} \backslash\{0\} . \\
& j_{1}, \ldots, j_{k_{1}+1} \in \mathcal{S}(\phi) \\
& k+j_{1}+\cdots+j_{k_{1}+1}=p
\end{aligned}
$$

- Let us show first that $\operatorname{Irr}(\phi)=\operatorname{Irr}(\breve{\phi})$. Using Lemma 5.1, we reason as in the one variable case. We must take into account that for $h \geqslant 2$, the element $r \in \mathbb{N}^{*}(\mathcal{S}(\breve{\phi})) \backslash \mathbb{N}^{*}(\mathcal{S}(\phi))$ chosen to be minimal with this property (for the componentwise partial order) is not necessarily unique. Let us choose $r$ to be the smallest element with this property, relative to an additive order $\leq$ dominating $\mathbb{Q}_{+}^{h}$. Then the proof of the assertion follows exactly by the same argument as for $h=1$.

- We prove the identities relating the coefficients associated to the irreducible exponents of $\phi$ and $\breve{\phi}$. If $r \in \operatorname{Irr}(\phi)=\operatorname{Irr}(\check{\phi})$ and if: $k+j_{1}+\cdots+j_{k_{1}+1}=r$ for $k \in \mathcal{S}(\check{\phi})$ and $j_{1}, \ldots j_{k_{1}+1} \in \mathcal{S}(\phi)$, then we obtain, by the same argument as in the one variable case, that $k=r$ or $k=0$. We deduce the following analogues of equation (3.12):

$$
[\check{\phi}]_{0}[\phi]_{r}+[\check{\phi}]_{r}[\phi]_{0}^{r_{1}+1}=0
$$

from which one gets the stated equality between coefficients of terms with irreducible exponents.

Combining Proposition 5.12 with Lemma 5.9, we obtain the following extension of Corollary 3.17

Corollary 5.13. Let $\phi \in \mathbb{K}\left[\left[t_{1}, t_{2}, \ldots, t_{h}\right]\right]^{*}, N \in \mathbb{N}^{*}$ and $M$ be any lattice of $\mathbb{Q}^{h}$. Then the sequences of essential exponents of $\phi, \phi^{N}$ and $\check{\phi}$ relative to $M$ coincide. 


\subsection{Newton-Puiseux series in several variables.}

We will consider the following analogue of the ring of Newton-Puiseux series in one variable:

$$
\mathbb{K}\left[\left[x_{1}^{1 / \mathbb{N}}, x_{2}^{1 / \mathbb{N}}, \ldots, x_{h}^{1 / \mathbb{N}}\right]\right]:=\bigcup_{n_{i} \in \mathbb{N}^{*}, 1 \leqslant i \leqslant h} \mathbb{K}\left[\left[x_{1}^{1 / n_{1}}, x_{2}^{1 / n_{2}}, \ldots, x_{h}^{1 / n_{h}}\right]\right] .
$$

We say that its elements are Newton-Puiseux series in the variables $x_{1}, \ldots, x_{h}$. The support $S(\eta)$ of such a series $\eta$ is a subset with bounded denominators of $\mathbb{Q}_{+}^{h}$.

Definition 5.14. Assume that $f\left(x_{1}, y_{1}, x_{2}, \ldots, x_{h}\right) \in \mathbb{K}\left[\left[x_{1}, y_{1}, x_{2}, \ldots, x_{h}\right]\right]$ has vanishing constant term. A Newton-Puiseux series of $f$ relative to $\left(x_{1}, x_{2}, \ldots, x_{h}\right)$ is a series:

$$
\psi \in \mathbb{K}\left[\left[x_{1}^{1 / \mathbb{N}}, x_{2}^{1 / \mathbb{N}}, \ldots, x_{h}^{1 / \mathbb{N}}\right]\right]
$$

such that $f\left(x_{1}, \psi, x_{2}, \ldots, x_{h}\right)=0$. The series $\psi$ is called $x_{1}$-dominating if it is of the form:

$$
a \cdot x_{1}^{\lambda}(1+\text { higher order terms })
$$

where $\lambda \in \mathbb{Q}_{+}^{*}$, and $a \in \mathbb{K}^{*}$. A representation of $\psi \in \mathbb{K}\left[\left[x_{1}^{1 / \mathbb{N}}, x_{2}^{1 / \mathbb{N}}, \ldots, x_{h}^{1 / \mathbb{N}}\right]\right]$ of the form:

$$
\psi=\eta\left(x_{1}^{1 / n_{1}}, x_{2}^{1 / n_{2}}, \ldots, x_{h}^{1 / n_{h}}\right) \text { with } \eta \in \mathbb{K}\left[\left[t_{1}, t_{2}, \ldots, t_{h}\right]\right]
$$

is called primitive if it is primitive in each variable separately in the sense of Definition 2.5

Example 5.15. The Newton-Puiseux series $x_{1}^{3 / 2}+x_{1}^{7 / 4} x_{2}^{1 / 2}-2 x_{1}^{2} x_{3}^{1 / 3}$ is $x_{1}$-dominating. But the series: $x_{1}^{1 / 2}+x_{2}^{1 / 2}$ and $x_{2}^{1 / 3}+x_{1} x_{2}^{2 / 3}$ are not $x_{1}$-dominating. Instead, the second one is $x_{2}$-dominating.

If $\psi \in \mathbb{K}\left[\left[x_{1}^{1 / \mathbb{N}}, \ldots, x_{h}^{1 / \mathbb{N}}\right]\right]$, then there is a series $f \in \mathbb{K}\left[\left[x_{1}, y_{1}, x_{2}, \ldots, x_{h}\right]\right]$ such that $f\left(x_{1}, \psi, x_{2}, \ldots, x_{h}\right)=$ 0 . Indeed, one can get such an $f$ in the ring $\mathbb{K}\left[\left[x_{1}, \ldots, x_{h}\right]\right]\left[y_{1}\right]$ (cf. Remark 2.4 in the 1-variable case):

Remark 5.16. Recall that for any $n \in \mathbb{N}^{*}$, we denote by $G_{n}$ the subgroup of $\left(\mathbb{K}^{*}, \cdot\right)$ consisting of the $n$-roots of unity. Consider $n_{1}, \ldots, n_{h} \in \mathbb{N}^{*}$. Let $\mathbb{K}\left(\left(x_{1}^{1 / n_{1}}, \ldots, x_{h}^{1 / n_{h}}\right)\right)$ be the fraction field of $\mathbb{K}\left[\left[x_{1}^{1 / n_{1}}, \ldots, x_{h}^{1 / n_{h}}\right]\right]$. The field extension $\mathbb{K}\left(\left(x_{1}, \ldots, x_{h}\right)\right) \subset \mathbb{K}\left(\left(x_{1}^{1 / n_{1}}, \ldots, x_{h}^{1 / n_{h}}\right)\right)$ is finite and Galois. Its Galois group is isomorphic to $G_{n_{1}} \times \cdots \times G_{n_{h}}$, acting on $\mathbb{K}\left(\left(x_{1}^{1 / n_{1}}, \ldots, x_{h}^{1 / n_{h}}\right)\right)$ by:

$$
\left(\left(\rho_{1}, \ldots, \rho_{h}\right), x_{1}^{a_{1} / n_{1}} \cdots x_{h}^{a_{h} / n_{h}}\right) \rightarrow \rho_{1}^{a_{1}} \cdots \rho_{h}^{a_{h}} \cdot x_{1}^{a_{1} / n_{1}} \cdots x_{h}^{a_{h} / n_{h}} .
$$

If $\psi \in \mathbb{K}\left[\left[x_{1}^{1 / n_{1}}, \ldots, x_{h}^{1 / n_{h}}\right]\right]$ is a Newton-Puiseux series, then the field extension $\mathbb{K}\left(\left(x_{1}, \ldots, x_{h}\right)\right) \subset$ $\mathbb{K}\left(\left(x_{1}, \ldots, x_{h}\right)\right)[\psi]$ is finite and its Galois group $G$ is isomorphic to the quotient of $G_{n_{1}} \times \cdots \times G_{n_{h}}$ by its subgroup formed by those elements which leave $\psi$ fixed. If $n=|G|$ and $\psi_{1}=\psi, \psi_{2}, \ldots, \psi_{n}$ are the different conjugates of $\psi$ under the action of the group $G$, then the polynomial:

$$
f=\prod_{j=1}^{n}\left(y-\psi_{j}\right) \in \mathbb{K}\left[\left[x_{1}^{1 / n_{1}}, \ldots, x_{h}^{1 / n_{h}}\right]\right][y]
$$

is invariant under the action of $G_{n_{1}} \times \cdots \times G_{n_{h}}$ on its coefficients. It follows that $f$ must belong to $\mathbb{K}\left[\left[x_{1}, \ldots, x_{h}\right]\right][y]$ and that $\psi$ is a Newton-Puiseux series relative to $f$.

Remark 5.17. Let $f \in \mathbb{K}\left[\left[x_{1}, \ldots, x_{h}\right]\right]\left[y_{1}\right]$ be an irreducible polynomial such that its discriminant $\Delta_{y_{1}} f$ is the product of a monomial and of a unit in the ring $\mathbb{K}\left[\left[x_{1}, \ldots, x_{h}\right]\right]$. Then, by the Jung-Abhyankar theorem, all the roots of $f$ are Newton-Puiseux series in the variables $x_{1}, \ldots, x_{h}$ (see [1]). Let us mention that the roots obtained in this way have special properties, for instance, the Newton-Puiseux series $x_{1}^{3 / 2}+x_{2}^{5 / 2}$ cannot be a root of the polynomial $f$ (see Lemma 5.24). Notice also that if the discriminant of $f$ is not of this form, the roots may not be expressible as Newton-Puiseux series in the variables $x_{1}, \ldots, x_{h}$. An example of this last phenomenon is the polynomial $f=x_{1}^{3}+x_{2}^{3}+y_{1}^{2}$. 


\subsection{The generalized Halphen-Stolz inversion theorem.}

In this subsection we assume that $\psi$ is a $x_{1}$-dominating Newton-Puiseux power series with primitive representation (see Definition 5.14):

$$
\psi=\eta\left(x_{1}^{1 / n_{1}}, x_{2}^{1 / n_{2}}, \cdots, x_{h}^{1 / n_{h}}\right) .
$$

Then the dominating term $a \cdot x_{1}^{\lambda}$ of $\psi$ satisfies:

$$
\lambda=\frac{m_{1}}{n_{1}}
$$

with $m_{1} \in \mathbb{N}^{*}$. The series $\eta$ is therefore of the form:

$$
\eta\left(t_{1}, t_{2}, \ldots, t_{h}\right)=a \cdot t_{1}^{m_{1}}(1+\text { higher order terms }),
$$

with $m_{1}>0$ and $a \in \mathbb{K}^{*}$. Let us choose an $m_{1}$-th root $\tilde{a} \in \mathbb{K}^{*}$ of $a$. Then, we have a unique $m_{1}$-th root $t_{1} \tilde{\eta}\left(t_{1}, t_{2}, \ldots, t_{h}\right) \in \mathbb{K}\left[\left[t_{1}, t_{2}, \ldots, t_{h}\right]\right]$ of $\eta$ :

$$
\eta\left(t_{1}, t_{2}, \ldots, t_{h}\right)=\left(t_{1} \tilde{\eta}\left(t_{1}, t_{2}, \ldots, t_{h}\right)\right)^{m_{1}},
$$

with constant term $[\tilde{\eta}]_{0}=\tilde{a} \neq 0$.

Example 5.18. Start from the Newton-Puiseux series: $\psi=x_{1}^{3 / 2}+x_{1}^{7 / 4} x_{2}^{1 / 2}-2 x_{1}^{2} x_{3}^{1 / 3}$. We get $a=1$, $n_{1}=4, n_{2}=2, n_{3}=3$ and $\psi=\eta\left(x_{1}^{1 / 4}, x_{2}^{1 / 2}, x_{3}^{1 / 3}\right)$ where $\eta(t)=t_{1}^{6}+t_{1}^{7} t_{2}-2 t_{1}^{8} t_{3}=t_{1}^{6}\left(1+t_{1} t_{2}-2 t_{1}^{2} t_{3}\right)$. This shows that $m_{1}=6$ and if $\tilde{a}=1$ then: $\tilde{\eta}(t)=\left(1+t_{1} t_{2}-2 t_{1}^{2} t_{3}\right)^{1 / 6}:=1+\sum_{k \in \mathbb{N} *}\left(\begin{array}{c}1 / 6 \\ k\end{array}\right)\left(t_{1} t_{2}-2 t_{1}^{2} t_{3}\right)^{k}$.

Let us come back to the general case. Denote by $\tilde{\xi}\left(u_{1}, t_{2}, \ldots, t_{h}\right) \in \mathbb{K}\left[\left[u_{1}, t_{2}, \ldots, t_{h}\right]\right]^{*}$ the dual series of $\tilde{\eta}\left(t_{1}, t_{2}, \ldots, t_{h}\right)$ with respect to $t_{1}$ (see Definition [5.10). Hence, one has the equivalence (see (5.2) ):

$$
u_{1}=t_{1} \tilde{\eta}\left(t_{1}, t_{2}, \ldots, t_{h}\right) \Leftrightarrow t_{1}=u_{1} \tilde{\xi}\left(u_{1}, t_{2}, \ldots, t_{h}\right)
$$

We know that there exists a series $f\left(x_{1}, y_{1}, x_{2}, \ldots, x_{h},\right) \in \mathbb{K}\left[\left[x_{1}, y_{1}, x_{2}, \ldots, x_{h}\right]\right]$ such that:

$$
f\left(x_{1}, \eta\left(x_{1}^{1 / n_{1}}, x_{2}^{1 / n_{2}}, \ldots, x_{h}^{1 / n_{h}}\right), x_{2}, \ldots, x_{h}\right)=0 .
$$

Replacing each $x_{i}$ by $t_{i}^{n_{i}}$ and using the equality (5.9), we get:

$$
f\left(t_{1}^{n_{1}},\left(t_{1} \tilde{\eta}\left(t_{1}, t_{2}, \ldots, t_{h}\right)\right)^{m_{1}}, t_{2}^{n_{2}}, \ldots, t_{h}^{n_{h}}\right)=0 .
$$

By doing the second change of variable of formula (5.10), we deduce that:

$$
f\left(\left(u_{1} \tilde{\xi}\left(u_{1}, t_{2}, \ldots, t_{h}\right)\right)^{n_{1}}, u_{1}^{m_{1}}, t_{2}^{n_{2}}, \ldots, t_{h}^{n_{h}}\right)=0 .
$$

Consequently, if one defines:

$$
\xi\left(u_{1}, t_{2}, \ldots, t_{h}\right)=\left(u_{1} \tilde{\xi}\left(u_{1}, t_{2}, \ldots, t_{h}\right)\right)^{n_{1}}
$$

(an equation which is analogous to (5.9)), then one sees that:

$$
f\left(\xi\left(y_{1}^{1 / m_{1}}, x_{2}^{1 / n_{2}}, \ldots, x_{h}^{1 / n_{h}}\right), y_{1}, x_{2}, \ldots, x_{h}\right)=0,
$$

that is, $\xi\left(y_{1}^{1 / m_{1}}, x_{2}^{1 / n_{2}}, \ldots, x_{h}^{1 / n_{h}}\right)$ is a Newton-Puiseux series of $f\left(x_{1}, y_{1}, x_{2}, \ldots, x_{h}\right)$ with respect to the variables $\left(y_{1}, x_{2}, \ldots, x_{h}\right)$ (see Definition [5.14).

Recall that we denote by $\left(\nu_{1}, \nu_{2}, \ldots, \nu_{h}\right)$ the canonical basis of $\mathbb{Q}^{h}$. The following lemma is a multivariable analogue of Lemma 4.3, formulated using Definition 5.3 .

Lemma 5.19. Let $\leq$ be an additive order of $\mathbb{Q}^{h}$ dominating $\mathbb{Q}_{+}^{h}$. Denote:

$$
\left\{\begin{array}{l}
\operatorname{ess}\left(\eta, n_{1} \mathbb{Z} \nu_{1}+\mathbb{Z} \nu_{2}+\cdots+\mathbb{Z} \nu_{h}, \preceq\right)=\left(m_{1} \nu_{1}, \epsilon_{1}, \ldots, \epsilon_{d}\right) \\
\operatorname{ess}\left(\xi, m_{1} \mathbb{Z} \nu_{1}+\mathbb{Z} \nu_{2}+\cdots+\mathbb{Z} \nu_{h}, \preceq\right)=\left(n_{1} \nu_{1}, \epsilon_{d}^{\prime}, \ldots, \epsilon_{d^{\prime}}^{\prime}\right)
\end{array}\right.
$$

Then:

$$
\left\{\begin{array}{l}
\operatorname{ess}\left(\tilde{\eta}, \operatorname{gcd}\left(n_{1}, m_{1}\right) \mathbb{Z} \nu_{1}+\mathbb{Z} \nu_{2}+\cdots+\mathbb{Z} \nu_{h}, \preceq\right)=\left(0, \epsilon_{1}-m_{1} \nu_{1}, \ldots, \epsilon_{d}-m_{1} \nu_{1}\right), \\
\operatorname{ess}\left(\tilde{\xi}, \operatorname{gcd}\left(n_{1}, m_{1}\right) \mathbb{Z} \nu_{1}+\mathbb{Z} \nu_{2}+\cdots+\mathbb{Z} \nu_{h}, \preceq\right)=\left(0, \epsilon_{1}^{\prime}-n_{1} \nu_{1}, \ldots, \epsilon_{d^{\prime}}^{\prime}-n_{1} \nu_{1}\right) .
\end{array}\right.
$$


Proof. By symmetry, we may treat only the case of the series $\tilde{\eta}$. As $\tilde{\eta} \in \mathbb{K}\left[\left[t_{1}, t_{2}, \ldots, t_{h}\right]\right]^{*}$, Proposition 5.12 implies that $\operatorname{Irr}(\tilde{\eta})=\operatorname{Irr}\left(\tilde{\eta}^{m}\right)$. Combining this with Lemma 5.9] we see that for any lattice $M$ of $\mathbb{Q}^{h}$ one has:

$$
\operatorname{ess}(\tilde{\eta}, M, \preceq)=\operatorname{ess}(\operatorname{Irr}(\tilde{\eta}), M, \preceq)=\operatorname{ess}\left(\operatorname{Irr}\left(\tilde{\eta}^{m}\right), M, \preceq\right)=\operatorname{ess}\left(\tilde{\eta}^{m}, M, \preceq\right)
$$

Thus, it is enough to prove that $\left(0, \epsilon_{1}-m_{1} \nu_{1}, \ldots, \epsilon_{d}-m_{1} \nu_{1}\right)$ is the sequence of essential exponents of $\tilde{\eta}^{m_{1}}$ relative to $\operatorname{gcd}\left(n_{1}, m_{1}\right) \mathbb{Z} \nu_{1}+\mathbb{Z} \nu_{2}+\cdots+\mathbb{Z} \nu_{h}$ and the chosen additive order. By formula (5.9), we get $\tilde{\eta}^{m_{1}}=t_{1}^{-m_{1}} \eta$. Therefore $\mathcal{S}\left(\tilde{\eta}^{m_{1}}\right)=\mathcal{S}(\eta)-m_{1} \nu_{1}$. Using Definition 5.5. we see that we are done if we prove that:

- $\min \left(\mathcal{S}(\eta)-m_{1} \nu_{1}\right)=0$.

- For all $k \in\{1, \ldots, d\}$ :

$\epsilon_{k}-m_{1} \nu_{1}=\min \left(\left(\mathcal{S}(\eta)-m_{1} \nu_{1}\right) \backslash\left(\operatorname{gcd}\left(n_{1}, m_{1}\right) \mathbb{Z} \nu_{1}+\mathbb{Z} \nu_{2}+\cdots+\mathbb{Z} \nu_{h}+\mathbb{Z}\left\{0, \epsilon_{1}-m_{1} \nu_{1}, \ldots, \epsilon_{k-1}-m_{1} \nu_{1}\right\}\right)\right)$.

- $\mathcal{S}(\eta)-m_{1} \nu_{1} \subset \operatorname{gcd}\left(n_{1}, m_{1}\right) \mathbb{Z} \nu_{1}+\mathbb{Z} \nu_{2}+\cdots+\mathbb{Z} \nu_{h}+\mathbb{Z}\left\{0, \epsilon_{1}-m_{1} \nu_{1}, \ldots, \epsilon_{d}-m_{1} \nu_{1}\right\}$,

where the minimum is taken with respect to the additive order $\leq$. But all these facts are immediate from the definition of the essential exponents $\epsilon_{i}$, because:

$$
\begin{gathered}
\operatorname{gcd}\left(n_{1}, m_{1}\right) \mathbb{Z} \nu_{1}+\mathbb{Z} \nu_{2}+\cdots+\mathbb{Z} \nu_{h}+\mathbb{Z}\left\{0, \epsilon_{1}-m_{1} \nu_{1}, \ldots, \epsilon_{k-1}-m_{1} \nu_{1}\right\}= \\
=n_{1} \mathbb{Z} \nu_{1}+\mathbb{Z} \nu_{2}+\cdots+\mathbb{Z} \nu_{h}+\mathbb{Z}\left\{m_{1} \nu_{1}, \epsilon_{1}, \ldots, \epsilon_{k-1}\right\}
\end{gathered}
$$

for all $1 \leqslant k \leqslant d$, an equality which may be proved immediately by double inclusion.

Our extension of Theorem 4.4 to the case of an arbitrary number of variables follows then exactly as in the one variable case:

Theorem 5.20. Let $\tilde{\eta} \in \mathbb{K}\left[\left[t_{1}, t_{2}, \ldots, t_{h}\right]\right]^{*}$ and $\tilde{\xi} \in \mathbb{K}\left[\left[u_{1}, t_{2}, \ldots, t_{h}\right]\right]^{*}$ be dual relative to the first coordinate and consider $m_{1}, n_{1} \in \mathbb{N}^{*}$. Let $\tilde{a}$ be the constant term of $\tilde{\eta}$. Denote by $\left(\nu_{1}, \nu_{2}, \ldots, \nu_{h}\right)$ the canonical basis of the free abelian group $\mathbb{Z}^{h}$. Introduce the $t_{1}$-dominating and $u_{1}$-dominating series:

$$
\left\{\begin{array}{l}
\eta\left(t_{1}, t_{2}, \ldots, t_{h}\right)=\left(t_{1} \tilde{\eta}\left(t_{1}, t_{2}, \ldots, t_{h}\right)\right)^{m_{1}}, \\
\xi\left(u_{1}, t_{2}, \ldots, t_{h}\right)=\left(u_{1} \tilde{\xi}\left(u_{1}, t_{2}, \ldots, t_{h}\right)\right)^{n_{1}},
\end{array}\right.
$$

and their sequences of essential exponents relative to an additive order $\leq$ dominating $\mathbb{Q}_{\geqslant 0}^{h}$ :

$$
\left\{\begin{array}{l}
\operatorname{ess}\left(\eta, n_{1} \mathbb{Z} \nu_{1}+\mathbb{Z} \nu_{2}+\cdots+\mathbb{Z} \nu_{h}, \leq\right)=\left(m_{1} \nu_{1}, \epsilon_{1}, \ldots, \epsilon_{d}\right) \\
\operatorname{ess}\left(\xi, m_{1} \mathbb{Z} \nu_{1}+\mathbb{Z} \nu_{2}+\cdots+\mathbb{Z} \nu_{h}, \leq\right)=\left(n_{1} \nu_{1}, \epsilon_{1}^{\prime}, \ldots, \epsilon_{d^{\prime}}^{\prime}\right)
\end{array}\right.
$$

Then one has the following inversion formulae for exponents and coefficients, where we denote by $\epsilon_{k, 1}$ the first coordinate of $\epsilon_{k} \in \mathbb{Q}_{+}^{h}$ (that is, the coefficient of $\nu_{1}$ in the expansion $\epsilon_{k}=\sum_{i=1}^{h} \epsilon_{k, i} \nu_{i}$ ):

$$
\begin{gathered}
d^{\prime}=d, \\
\epsilon_{k}^{\prime}+m_{1} \nu_{1}=\epsilon_{k}+n_{1} \nu_{1} \text {, for all } k \in\{1, \ldots, d\}, \\
{[\xi]_{n}=\tilde{a}^{-n_{1}} \quad \text { and }[\xi]_{\epsilon_{k}^{\prime}}=-\frac{n_{1}}{m_{1}} \tilde{a}^{-n_{1}-\epsilon_{k, 1}}[\eta]_{\epsilon_{k}} \text {, for all } k \in\{1, \ldots, d\} .}
\end{gathered}
$$

As a consequence of this theorem, we get the following generalization of the Halphen-Stolz inversion theorem (Corollary 4.5):

\section{Corollary 5.21 (The generalized Halphen-Stolz inversion theorem).}

Let $\eta\left(x_{1}^{1 / n_{1}}, x_{2}^{1 / n_{2}}, \ldots, x_{h}^{1 / n_{h}}\right)$ and $\xi\left(y_{1}^{1 / m_{1}}, x_{2}^{1 / n_{2}}, \ldots, x_{h}^{1 / n_{h}}\right)$ be Newton-Puiseux series of $f\left(x_{1}, y_{1}, x_{2}, \ldots, x_{h}\right)$ relative to $\left(x_{1}, x_{2}, \ldots, x_{h}\right)$ and $\left(y_{1}, x_{2}, \ldots, x_{h}\right)$ respectively. As before, we assume that $\eta\left(t_{1}, t_{2}, \ldots, t_{h}\right)=$ $\left(t_{1} \tilde{\eta}\left(t_{1}, t_{2}, \ldots, t_{h}\right)\right)^{m_{1}}$ and $\xi\left(u_{1}, t_{2}, \ldots, t_{h}\right)=\left(u_{1} \tilde{\xi}\left(u_{1}, t_{2}, \ldots, t_{h}\right)\right)^{n_{1}}$, where $\tilde{\eta}\left(t_{1}, t_{2}, \ldots, t_{h}\right)$ and $\tilde{\xi}\left(u_{1}, t_{2}, \ldots, t_{h}\right)$ are dual relative to the first coordinate and $[\tilde{\eta}]_{0}=\tilde{a}$. Let $\leq$ be a fixed additive order dominating $\mathbb{Q}_{+}^{h}$. Denote:

$$
\left\{\begin{array}{l}
\operatorname{ess}\left(\eta\left(x_{1}^{1 / n_{1}}, x_{2}^{1 / n_{2}}, \ldots, x_{h}^{1 / n_{h}}\right), \mathbb{Z}^{h}, \leq\right)=\left(\frac{m_{1}}{n_{1}} \nu_{1}, e_{1}, \ldots, e_{d}\right) \\
\operatorname{ess}\left(\xi\left(y_{1}^{1 / m_{1}}, x_{2}^{1 / n_{2}}, \ldots, x_{h}^{1 / n_{h}}\right), \mathbb{Z}^{h}, \leq\right)=\left(\frac{n_{1}}{m_{1}} \nu_{1}, e_{1}^{\prime}, \ldots, e_{d^{\prime}}^{\prime}\right)
\end{array}\right.
$$


Then one has the following inversion formulae for exponents and coefficients, where we denote by $e_{k, 1}$ the first coordinate of $e_{k} \in \mathbb{Q}_{+}^{h}$ (that is, the coefficient of $\nu_{1}$ in the expansion $e_{k}=\sum_{i=1}^{h} e_{k, i} \nu_{i}$ ):

$$
\begin{aligned}
& \quad d^{\prime}=d . \\
& (5.19) \\
& (5.20) \\
& {\left[\xi\left(y_{1}^{1 / m_{1}}, \ldots\right)\right]_{n_{1} / m_{1}}=\tilde{a}^{-n_{1}} \text { and }\left[\xi\left(y_{1}^{1 / m_{1}}, \ldots\right)\right]_{e_{k}^{\prime}}^{\prime}=-\frac{n_{1}}{m_{1}} \tilde{a}^{-\left(1+e_{k, 1}\right) n_{1}}\left[\eta\left(x_{1}^{1 / n_{1}}, \ldots\right)\right]_{e_{k}} \text { for all } k \in\{1, \ldots, d\} .}
\end{aligned}
$$

In the case in which $\tilde{a}=1$, the inversion formula for the coefficients stated in Corollary 5.21 may be written in a more symmetric way, easier to remember, and analogous to Corollary 4.6

Corollary 5.22. Assume moreover that the constant coefficient $\tilde{a}$ of $\tilde{\eta}$ is equal to 1 . Then:

$$
[\xi]_{n_{1} / m_{1}}=1=[\eta]_{m_{1} / n_{1}}, \quad \text { and } \quad m_{1}[\xi]_{\epsilon_{k}^{\prime}}+n_{1}[\eta]_{\epsilon_{k}}=0 \text { for all } k \in\{1, \ldots, d\} .
$$

In order to summarize our reasoning, let us draw the analogue of the flow-chart (4.17) in which $f\left(x_{1}, y_{1}, x_{2}, \ldots, x_{h}\right) \in \mathbb{K}\left[\left[x_{1}, y_{1}, x_{2}, \ldots, x_{h}\right]\right]$ is an irreducible series:

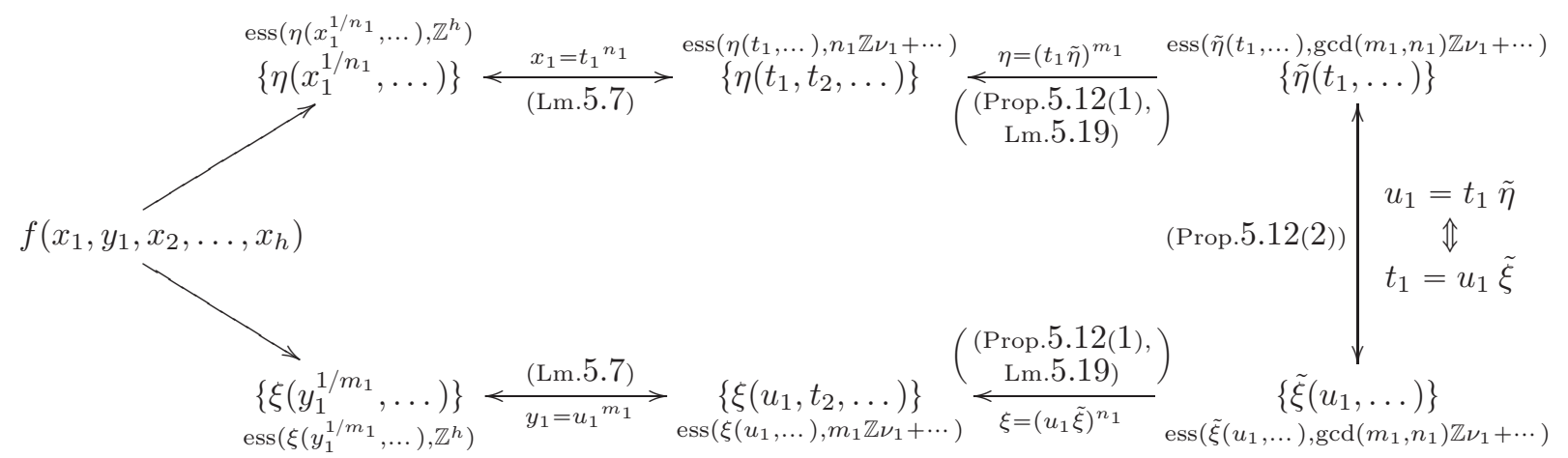

\subsection{The special case of quasi-ordinary series.}

Among the Newton-Puiseux series in several variables, the quasi-ordinary ones form a distinguished subclass, having many special properties. We compare both classes in this section with the help of additive orders and toric modifications. In particular, we get that Lipman's inversion theorem for quasi-ordinary series can be seen as a particular case of our generalized inversion theorem (Corollary 5.21).

Definition 5.23. A series $\psi \in \mathbb{K}\left[\left[x_{1}^{1 / \mathbb{N}}, \ldots, x_{h}^{1 / \mathbb{N}}\right]\right]$ is quasi-ordinary if $\psi$ is a Newton-Puiseux series relative to an irreducible polynomial $f \in \mathbb{K}\left[\left[x_{1}, \ldots, x_{h}\right]\right]\left[y_{1}\right]$, such that the discriminant, $\Delta_{y_{1}}(f) \in$ $\mathbb{K}\left[\left[x_{1}, \ldots, x_{h}\right]\right]$ of $f$ with respect to $y_{1}$, is the product of a monomial and of a unit in the ring $\mathbb{K}\left[\left[x_{1}, \ldots, x_{h}\right]\right]$.

If the discriminant $\Delta_{y_{1}}(f)$ of $f \in \mathbb{K}\left[\left[x_{1}, \ldots, x_{d}\right]\right]\left[y_{1}\right]$ is a monomial times a unit then, by the JungAbhyankar theorem (see [1] and [22]), $f$ factors in the ring $\mathbb{K}\left[\left[x_{1}^{1 / \mathbb{N}}, \ldots, x_{h}^{1 / \mathbb{N}}\right]\right]\left[y_{1}\right]$ as a product of polynomials of degree 1 in the variable $y_{1}$. If $y_{1}-\psi, y_{1}-\psi^{\prime}$ are two different factors of $f$ in this ring, then $\psi-\psi^{\prime}$ divides the discriminant $\Delta_{y_{1}}(f)$, hence $\psi-\psi^{\prime}$ is the product of a monomial times a unit in the $\operatorname{ring} \mathbb{K}\left[\left[x_{1}^{1 / \mathbb{N}}, \ldots, x_{h}^{1 / \mathbb{N}}\right]\right]$. The monomials obtained in this way:

$$
x_{1}^{\alpha_{k, 1}} \ldots x_{h}^{\alpha_{k, h}} \text {, for } k \in\{1, \ldots, g\},
$$

are called the characteristic monomials, and the tuples:

$$
\alpha_{k}=\left(\alpha_{k, 1}, \ldots, \alpha_{k, h}\right) \text {, for } k \in\{1, \ldots, g\},
$$

the characteristic exponents of the quasi-ordinary series $\psi$. Lipman showed that the characteristic exponents determine many features of the geometry of the germ of hypersurface defined by $f$ (for precise 
definitions and related results, see for instance [24, 26, 27, 14]). He also proved the following combinatorial characterization of quasi-ordinary power series (see [24, Proposition 1.5] and [14, Proposition 1.3]):

Lemma 5.24. Denote by $\leqslant$ the coordinate-wise order on $\mathbb{Q}^{h}$. A series $\psi \in \mathbb{K}\left[\left[x_{1}^{1 / \mathbb{N}}, \ldots, x_{h}^{1 / \mathbb{N}}\right]\right]$ is quasiordinary if and only if there exist an integer $n \geqslant 1$ and elements $\lambda_{1}, \ldots, \lambda_{r} \in \mathcal{S}(\psi)$ such that:

(1) The support $\mathcal{S}(\psi)$ is included in $\frac{1}{n} \mathbb{Z}_{+}^{h}$.

(2) Every $\lambda \in \mathcal{S}(\psi)$ belongs to the group $\mathbb{Z}^{h}+\sum_{\lambda_{j} \leqslant \lambda} \mathbb{Z} \lambda_{j}$.

(3) $\lambda_{i} \leqslant \lambda_{i+1}$, for every $i \in\{1, \ldots, r-1\}$.

(4) $\lambda_{i}$ does not belong to the group $\mathbb{Z}^{h}+\sum_{j \leqslant i-1} \mathbb{Z} \lambda_{j}$, for every $i \in\{1, \ldots, r\}$.

If such elements exist, then they are the characteristic exponents of $\psi$.

The following lemma is an analogue of Lemma 3.13 Its proof is a consequence of the definitions of essential exponents and of Lemma 5.24. It shows how to recover the characteristic exponents of a quasi-ordinary series from a sequence of essential exponents relative to the lattice $\mathbb{Z}^{h}$ and any additive order $\leq$ dominating $\mathbb{Q}_{+}^{h}$.

Lemma 5.25. Let $\psi \in \mathbb{K}\left[\left[x_{1}^{1 / \mathbb{N}}, \ldots, x_{h}^{1 / \mathbb{N}}\right]\right]$ be a quasi-ordinary series with characteristic exponents $\alpha_{1}, \ldots, \alpha_{g}$. Let us denote by $\left(e_{0}, \ldots, e_{d}\right)$ the sequence of essential elements of the support $S(\psi)$ relative to the lattice $\mathbb{Z}^{h}$ and a fixed additive order $\leq$ dominating $\mathbb{Q}_{+}^{h}$. Then:

- If $e_{0} \notin \mathbb{Z}^{h}$, then $g=d+1$ and $\alpha_{k}=e_{k-1}$ for $k \in\{1, \ldots, d+1\}$.

- If $e_{0} \in \mathbb{Z}^{h}$, then $g=d$ and $\alpha_{k}=e_{k}$ for $k \in\{1, \ldots, d\}$.

Remark 5.26. Lipman proved an inversion theorem for the characteristic exponents of a quasi-ordinary series $\psi$, when $\psi$ is $x_{1}$-dominant. This result appeared in Lipman's PhD Thesis 24, Lemma 2.3 and table 4.4], see also [26, while its proof was published later in [28. This proof is written in the two variable case but it extends naturally to more variables. See also [17, Proposition 5.5]. Thanks to Corollary 5.21 and Lemma 5.25. we see that Lipman's inversion theorem for quasi-ordinary series is a particular case of the part concerning exponents of our inversion theorem (Corollary 5.21) for $x_{1}$-dominant Newton-Puiseux series in several variables.

We end this paper with some remarks relating geometrically the Newton-Puiseux series with the quasiordinary series by using methods of toric geometry. They are inspired by the second-named author's proof of [16, Théorème 3].

Let $f \in \mathbb{K}\left[\left[x_{1}, \ldots, x_{h}\right]\right]\left[y_{1}\right]$ be a reduced polynomial such that $f(0, \ldots, 0)=0$. Assume that $f$ is not quasi-ordinary. Then, the discriminant $\Delta_{y_{1}}(f) \in \mathbb{K}\left[\left[x_{1}, \ldots, x_{h}\right]\right]$ is not of the form a monomial times a unit. It follows that the dual fan associated to the Newton polyhedron of $\Delta_{y_{1}}(f)$ defines a nontrivial subdivision of the positive quadrant $\left(\mathbb{R}^{h}\right)_{+}^{\vee}$ of the vector space $\left(\mathbb{R}^{h}\right)^{\vee}$ of real weights of monomials $x_{1}^{k_{1}} \cdots x_{h}^{k_{h}}$. Let $\Sigma$ be a regular subdivision of this dual fan. One has an associated toric modification $X_{\Sigma} \rightarrow \mathbb{K}^{h}$, which is obtained by patching the monomial maps associated to the $h$-dimensional cones of $\Sigma$. See for instance [15] or [16 for the basic definitions used in these methods of toric geometry.

Let $\sigma \in \Sigma$ be a $h$-dimensional cone of the fan $\Sigma$. It is spanned by the forms $\gamma_{1}, \ldots, \gamma_{h}$, which are the primitive lattice vectors of the lattice $\left(\mathbb{Z}^{h}\right)^{\vee}$ lying on the edges of the cone $\sigma$. By the definition of the dual fan, the following property holds:

Lemma 5.27. All the forms $\gamma_{1}, \ldots, \gamma_{h}$ reach their minimum value on the support of the discriminant $\Delta_{y_{1}}(f)$ at the same vertex $\lambda_{0}$ of its Newton polyhedron.

We consider the coordinates $\left(\gamma_{s, 1}, \ldots, \gamma_{s, h}\right)$ of the vectors $\gamma_{s}, s \in\{1, \ldots, h\}$, with respect to the dual basis of $\nu_{1}, \ldots, \nu_{h}$. Let $q_{\sigma} \in \mathrm{GL}(h, \mathbb{Q})$ be the linear map defined, with respect to the canonical basis $\nu_{1}, \ldots, \nu_{h}$ of $\mathbb{Q}^{h}$, by the matrix whose rows are $\left(\gamma_{s, 1}, \ldots, \gamma_{s, h}\right)$, for every $s \in\{1, \ldots, h\}$.

The monomial map

$$
\mathbb{K}\left[x_{1}, \ldots, x_{h}\right] \rightarrow \mathbb{K}\left[v_{1}, \ldots, v_{h}\right], \quad x^{\lambda} \rightarrow v^{q_{\sigma}(\lambda)}
$$


defines the chart $\mathbb{K}_{\sigma}^{h} \rightarrow \mathbb{K}^{h}$ of the toric modification of $X_{\Sigma} \rightarrow \mathbb{K}^{h}$, associated to cone $\sigma$. If:

$$
F=\sum_{\gamma=(\alpha, \beta) \in \mathbb{N}^{h} \times \mathbb{N}} c_{\gamma} x^{\alpha} y_{1}^{\beta} \in \mathbb{K}\left[\left[x_{1}, \ldots, x_{h}, y_{1}\right]\right]
$$

then the pull-back of $F$ on $\mathbb{K}_{\sigma}^{h} \times \mathbb{K}$ is defined by:

$$
F_{\sigma}=\sum_{\gamma=(\alpha, \beta) \in \mathbb{N}^{h} \times \mathbb{N}} c_{\gamma} v^{q_{\sigma}(\alpha)} y_{1}^{\beta} \in \mathbb{K}\left[\left[v_{1}, \ldots, v_{h}, y_{1}\right]\right] .
$$

Assume that $\psi=\sum c_{\lambda} x^{\lambda} \in \mathbb{K}\left[\left[x_{1}^{1 / \mathbb{N}}, \ldots, x_{h}^{1 / \mathbb{N}}\right]\right]$ is a Newton-Puiseux series of $f$. Then, by definition $\psi_{\sigma}:=\sum c_{\lambda} v^{q(\lambda)} \in \mathbb{K}\left[\left[v_{1}^{1 / \mathbb{N}}, \ldots, v_{h}^{1 / \mathbb{N}}\right]\right]$ is a Newton-Puiseux series of the pull-back $f_{\sigma}$ of $f$. In addition, $q\left(\lambda_{0}\right)$ belongs to the support of the discriminant $\Delta_{y_{1}}\left(f_{\sigma}\right)$. By Lemma 5.27 if $q(\lambda)$ belongs to the support of $\Delta_{y_{1}}\left(f_{\sigma}\right)$, then $q\left(\lambda_{0}\right) \leqslant q(\lambda)$ (for the coordinate-wise order). This implies that the Newton-Puiseux series $\psi_{\sigma}$ is quasi-ordinary, since the discriminant $\Delta_{y_{1}}\left(f_{\sigma}\right)$ is of the form $v^{q_{\sigma}\left(\lambda_{0}\right)}$ times a unit. Using Lemma 5.7 and the fact that $\sigma$ is a regular cone (which implies that $q_{\sigma}\left(\mathbb{Z}^{h}\right)=\mathbb{Z}^{h}$ ), we obtain also the relation:

$$
q_{\sigma}\left(\operatorname{ess}\left(\psi, \mathbb{Z}^{h}, \leq_{q_{\sigma}}\right)\right)=\operatorname{ess}\left(\psi_{\sigma}, \mathbb{Z}^{h}, \leq\right)
$$

between the essential exponents of $\psi$ and the essential exponents of the quasi-ordinary series $\psi_{\sigma}$.

Example 5.28. By Lemma [5.24, the series $\psi=x_{1}^{3 / 2}+x_{2}^{1 / 4}+x_{1}^{7 / 2} x_{2}^{5 / 2}$ is not quasi-ordinary. If we consider the chart of the blowing up of $0 \in \mathbb{K}^{2}$ given by $x_{1}=v_{1} v_{2}$ and $x_{2}=v_{2}$, whose associated cone we denote by $\sigma$, then we obtain the series:

$$
\psi_{\sigma}=v_{1}^{3 / 2} v_{2}^{3 / 2}+v_{2}^{1 / 4}+v_{1}^{7 / 2} v_{2}^{6} .
$$

By Lemma 5.24 the series $\psi_{\sigma}$ is quasi-ordinary. It has essential exponents $(0,1 / 4)$ and $(3 / 2,3 / 2)$ with respect to the lattice $\mathbb{Z}^{2}$ and any additive order $\leq$ of $\mathbb{Q}^{2}$ dominating $\mathbb{Q}_{+}^{2}$. By Lemma 5.25 these pairs are also the characteristic exponents of $\psi_{\sigma}$. By (5.23), the pairs $(0,1 / 4)$ and $(3 / 2,0)$ are the essential exponents of $\psi$ with respect to the order $\leq_{q_{\sigma}}$.

Remark 5.29. Tornero studied in 37 a notion of distinguished exponents of the Newton-Puiseux series $\psi \in \mathbb{K}\left[\left[x_{1}^{1 / \mathbb{N}}, \ldots, x_{h}^{1 / \mathbb{N}}\right]\right]$ with respect to a fixed additive order $\leq^{\prime}$ of $\mathbb{N}^{d}$. In Example [5.28 one can check that the distinguished exponents of $\psi$ relative to the additive order $\leq_{q_{\sigma}}$ correspond to the characteristic exponents of the quasi-ordinary series $\psi_{\sigma}$. One can prove that this is a general phenomenon. By the previous discussion, it is enough to show that given a fixed additive order $\leq^{\prime}$, there exists a unique $h$-dimensional cone $\sigma \in \Sigma$ and a unique additive order $\leq$ dominating $\mathbb{Q}_{+}^{h}$ such that the orders $\leq^{\prime}$ and $\leq_{q_{\sigma}}$ coincide. Indeed, the cone $\sigma$ is the unique $h$-dimensional cone of $\Sigma$ such that the additive order $\leq^{\prime}$ dominates $\sigma^{\vee} \cap \mathbb{Q}^{h}$, where $\sigma^{\vee}$ is the dual cone of $\sigma$ (the existence of $\sigma$ is a consequence of the properties of the Zariski-Riemann space of the fan $\Sigma$, see [12] and [18, Section 3.5]).

\section{REFERENCES}

[1] Abhyankar, S. S. On the ramification of algebraic functions. American Journal of Maths. 77 (1955), 575-592.

[2] Abhyankar, S. S. Inversion and invariance of characteristic pairs. American Journal of Maths. 89 (1967), $363-372$.

[3] Abhyankar, S. S. Inversion and invariance of characteristic terms: Part I. In The legacy of Alladi Ramakrishnan in the mathematical sciences. 93-168, Springer, New York, 2010.

[4] Borodzik, M. Puiseux expansion of a cuspidal singularity. Bulletin Polish Acad. Science 60 (2012), 21-25.

[5] Campillo, A. On saturations of curve singularities (any characteristic). Singularities, Part 1 (Arcata, Calif., 1981), 211-220, Proc. Sympos. Pure Math. 40, Amer. Math. Soc., Providence, RI, 1983.

[6] Campillo, A. Arithmetical aspects of saturation of singularities. Singularities (Warsaw, 1985), 121-137, Banach Center Publ. 20, PWN, Warsaw, 1988.

[7] Casas-Alvero, E. Singularities of plane curves. London Mathematical Society Lecture Note Series 276. Cambridge University Press, 2000

[8] Cox, D., Little, J., O'Shea, D. Ideals, varieties, and algorithms. Third Ed., Springer, 2007.

[9] Cutkosky, S. D. Resolution of singularities. Graduate Studies in Maths. 63. American Math. Society, 2004.

[10] de Jong, T., Pfister, G. Local analytic geometry. Advanced Lectures in Mathematics. Friedr. Vieweg \& Sohn, 2000.

[11] Enriques, F., Chisini, O. Lezioni sulla teoria geometrica delle equazioni e delle funzioni algebriche II. Zanichelli, Bologna, 1917. 
[12] Ewald, G., Ishida, M-N. Completion of real fans and Zariski-Riemann spaces, Tohôku Math. J., (2), 58, (2006), 189-218.

[13] Fischer, G. Plane algebraic curves. Student Mathematical Library 15. American Mathematical Society, 2001.

[14] Gau, Y.-N., Embedded topological classification of quasi-ordinary singularities, Memoirs Amer. Math. Soc. 388, (1988), 109-129.

[15] Goldin, R., Teissier, B. Resolving singularities of plane analytic branches with one toric morphism. Resolution of singularities (Obergurgl, 1997), 315-340, Progr. Math. 181, Birkhuser, Basel, 2000.

[16] González Pérez, P. D. Singularités quasi-ordinaires toriques et polyèdre de Newton du discriminant. Canad. J. Math. 52 (2000), no. 2, 348-368.

[17] González Pérez, P. D. The semigroup of a quasi-ordinary hypersurface. J. Inst. Math. Jussieu 2 (2003), no. 3, 383-399.

[18] González Pérez, P.D., Teissier, B. Toric geometry and the Semple-Nash modification Rev. R. Acad. Cienc. Exactas Fís. Nat. Ser. A Math. 08 (2014), no. 1, 1-48.

[19] Griffiths, P. Variations on a theorem of Abel. Inv. Math. 35 (1976), 321-390.

[20] Halphen, G. Sur une série de courbes analogues aux développées. Journal de maths. pures et appliquées (de Liouville) 3e série, tome 2 (1876), 87-144.

[21] Halphen, G. Étude sur les points singuliers des courbes algébriques planes. Appendix to G. Salmon's book Traité de géométrie analytique (courbes planes). Gauthier-Villars, Paris, 1884, 537-648.

[22] Jung, H. W. E. Darstellung der Funktionen eines algebraischen Körpers zweier unabhängigen Veränderlichen $x, y$ in der Umgebung einer stelle $x=a, y=b$. J. Reine Angew. Math. 133 (1908), 289-314.

[23] Lagrange, J.-L. Nouvelle méthode pour résoudre les équations littérales par le moyen des séries. Mémoires de l'Académie Royale des Sciences et Belles-Lettres de Berlin 24 (1770), 251-326.

[24] Lipman, J. Quasi-ordinary singularities of embedded surfaces. PhD Thesis, Harvard Univ., 1965. Available at: https://www.math.purdue.edu/ lipman/papers-older/

[25] Lipman, J. Relative Lipschitz-saturation. Amer. J. Math. 97 (1975), no. 3, 791-813.

[26] Lipman, J. Quasi-ordinary singularities of surfaces in $\mathbb{C}^{3}$, Proceedings of Symposia in Pure Mathematics 40, Part 2 (1983), 161-172.

[27] Lipman, J. Topological invariants of quasi-ordinary singularities. Mem. Amer. Math. Soc. 74 (1988), 388, 1-107.

[28] Lipman, J. Appendix to Gau, Y.-N. Embedded topological classification of quasi-ordinary Singularities. Memoirs Amer.Math.Soc. 388, (1988), 109-129.

[29] Newton, I. The method of fluxions and infinite series. Printed by H. Woodfall and sold by J. Nourse, London, 1736. Translated into french by M. Buffon, Debure libraire, 1740: La méthode des fluxions et des suites infinies.

[30] Pham P., Teissier B. Saturation Lipschitzienne d'une algèbre analytique complexe et saturation de Zariski. Prépublication École Polytechnique 1969. Also available at http://hal.archives-ouvertes.fr/hal-00384928/fr/

[31] Popescu-Pampu, P. Approximate roots. In Valuation theory and its applications. F.V. Kuhlmann et al. eds. Fields Inst. Communications 33, AMS 2003, 285-321.

[32] Puiseux, V. Recherches sur les fonctions algébriques. Journal de maths. pures et appliquées (de Liouville) 15 (1850), 365-480.

[33] Robbiano, L. On the theory of graded structures J. Symbolic Comput. 2, 2, (1986), 139-170.

[34] Smith, H.J.S. On the higher singularities of plane curves. Proc. London Math. Soc. 6 (1874), 153-182.

[35] Stanley, R. Enumerative combinatorics II. Cambridge Univ. Press, 1999.

[36] Stolz, O. Die Multiplicität der Schnittpunkte zweier algebraischer Curven. Math. Annalen 15 (1879), 122-160.

[37] Tornero, J. M. On Kummer extensions of the power series field. Math. Nachr. 281 no. 10, (2008), 1511-1519.

[38] Wall, C. T. C. Singular points of plane curves. London Math. Society Student Texts 63. Cambridge Univ. Press, 2004.

[39] Zariski, O. Algebraic surfaces. Springer-Verlag, 1935. A second supplemented edition appeared in 1971.

[40] Zariski, O. Studies in equisingularity III. Saturation of local rings and equisingularity. Amer. J. Math. 90 (1968), 961-1023.

[41] Zariski, O. Le problème des modules pour les branches planes. Avec un appendice de Bernard Teissier. Deuxième édition, Hermann, Paris, 1986. An English translation by Ben Lichtin was published in 2006 by the AMS with the title The moduli problem for plane branches.

Departamento de Matemáticas, Estadística e i.O. Sección de Matemáticas, Universidad de La Laguna. Apartado de Correos 456. 38200 La Laguna, Tenerife, España.

E-mail address: ergarcia@ull.es

Instituto de Ciencias Matemáticas (CSiC-UAM-UC3M-UCM), Departamento de Álgebra, Facultad de Ciencias Matemáticas, Universidad Complutense de Madrid, Plaza de las Ciencias 3, Madrid 28070, España.

E-mail address: pgonzalez@mat.ucm.es

Univ. Lille, UMr 8524, Laboratoire Paul Painlevé, F-59000 Lille, France.

E-mail address: patrick.popescu@math.univ-lille1.fr 